\title{
Disturbance Observer-Based Nonsingular Terminal Sliding Mode Control for Spacecraft Electromagnetic Docking
}

\author{
Jinghui Zhang $\mathbb{D}^{1}{ }^{1}$ Guoqiang Zeng $\mathbb{D}^{2}$, and Shifeng Zhang ${ }^{1}$ \\ ${ }^{1}$ College of Aerospace Science and Engineering, National University of Defense Technology, Changsha 410073, China \\ ${ }^{2}$ School of Remote Sensing and Information Engineering, Wuhan University, Wuhan 430072, China \\ Correspondence should be addressed to Guoqiang Zeng; gq.zeng@whu.edu.cn
}

Received 4 September 2020; Revised 24 September 2020; Accepted 30 September 2020; Published 28 October 2020

Academic Editor: James D. Biggs

Copyright (C) 2020 Jinghui Zhang et al. This is an open access article distributed under the Creative Commons Attribution License, which permits unrestricted use, distribution, and reproduction in any medium, provided the original work is properly cited.

\begin{abstract}
This paper presents a novel nonlinear sliding mode control scheme that combines on-line model modification, a nonlinear sliding mode controller, and a disturbance observer to solve the essential problems in spacecraft electromagnetic docking control, such as model uncertainties, unknown external disturbances, and inherent strong nonlinearity and coupling. An improved far-field model of electromagnetic force which is much more accurate than the widely used far-field model is proposed to enable the model parameters to be on-line self-adjusting. Then, the relationship between magnetic moment allocation and energy consumption is derived, and the optimal direction of the magnetic moment vector is obtained. Based on the proposed improved far-field model and the research results of magnetic moment allocation law, a fast-nonsingular terminal mode controller driven by a disturbance observer is designed in the presence of model uncertainties and external disturbances. The proposed control method is guaranteed to be chattering-free and to possess superior properties such as finite-time convergence, high-precision tracking, and strong robustness. Two simulation scenarios are conducted to illustrate the necessity of modifying the far-field model and the effectiveness of the proposed control scheme. The simulation results indicate the realization of electromagnetic soft docking and validate the merits of the proposed control scheme. In the end of this paper, some conclusions are drawn.
\end{abstract}

\section{Introduction}

Spacecraft relative motion control using intersatellite electromagnetic interaction is a technical innovation and research hotspot in the field of spacecraft control. As a typical noncontact internal force, the intersatellite electromagnetic force produced by the interaction of the electromagnetic mechanisms on two spacecraft has many distinct advantages over the traditional thrusters, such as the consumption of renewable energy rather than propellant, no plume contamination, and continuous and reversible controllability, and thus shows broad application prospects [1]. However, due to the relative motions of spacecraft and electromagnetic characteristics, the electromagnetic docking dynamics is inherently highly nonlinear and strongly coupled [2]. Moreover, because of the effects of the Earth's magnetic field, external perturbations, and imprecise modeling, there exist unknown external disturbances and model uncertainties that make it more difficult to realize superior docking performance [3-5].

The major applications of spacecraft relative motion control using electromagnetic interactions are to electromagnetic formation flight (EMFF), docking, on-orbit assembly [6], configuration restructuring [7], etc. All these applications involve electromagnet-based control and similar dynamics characteristics. Over the past decade, many researches have been conducted on EMFF [8-10], and their achievements can serve as a basis for the exploration of electromagnetic docking. However, the essential difference between EMFF and electromagnetic docking is the interaction distance between electromagnetic mechanisms, which leads to different dynamics models and control strategies. The relative distances among spacecraft in EMFF range from kilometers to hundreds of kilometers, but the relative distance between docking spacecraft is usually meter-sized. The exact 
electromagnetic force model based on electromagnetic theory cannot be solved analytically, and numerical integrator methods must be employed, which makes it difficult to design the controller. Moreover, the required numerical integration is computationally costly, particularly for small relative distances. The far-field model [11, 12], derived from the exact model using Taylor expansion, has been widely used due to its simple form and inexpensive computation. However, the error of the far-field model increases rapidly with decreasing relative distance; the percent error of the far-field model is less than $10 \%$ only when the ratio of relative distance to coil radius is larger than 6-8 [13]. Thus, the farfield model is usually used in EMFF rather than docking. To obtain an analytical model of the force between two electromagnets over a small distance, [14-16] present the analytical equations for the forces between coaxial and noncoaxial thin coils, but the forms are complex. Therefore, an analytical electromagnetic force model which lends itself to electromagnetic docking control should be established beforehand.

Spacecraft electromagnetic docking is an important research interest that is a fundamental technology for some space missions [17-19]. And recent researches on electromagnetic docking mostly focus on microsatellites that are attractive to high-risk, multiagent, or simple space missions because of their low cost, light weight, and mass production. In [6], dockings were classified according to their docking velocity as ultrasoft docking, soft docking, and hard docking if their relative velocity at docking is less than $1 \mathrm{~mm} / \mathrm{s}$, less than $1 \mathrm{~cm} / \mathrm{s}$, and above $1 \mathrm{~cm} / \mathrm{s}$, respectively. Given the reusability and safety of spacecraft, soft docking with nonfragility is usually required $[20,21]$. The magnet-based actuators are of three basic types: electromagnets, permanent magnets, and tethered electromagnets [22]. The permanent magnets usually provide a hard dock and are difficult to control but simple to implement. Tethered electromagnetic docking features tolerate failure but complexity in design. In EMFF, electromagnets, such as coils, are mainly used. Though the electromagnetic force decreases rapidly with increasing relative distance, high-temperature superconductors show promise in producing enough interaction for EMFF [9]. For microsatellite docking, due to the limited performance and close-range operation, using ordinary coils instead of superconductors may be feasible.

The existing researches on electromagnetic docking are mostly electromagnetic docking control methods and ground experiment tests [6]. However, there are still many issues to be solved. In practice, spacecraft electromagnetic docking is usually a trajectory tracking control problem and a high-risk operation at close range; thus, the robustness, high-precision control, and fast convergence rate are highly desirable [23]. Shi et al. proposed a disturbance observer-based controller with very good performance, which has been applied in spacecraft electromagnetic docking [24] and attitude control [25] in the presence of external disturbances and fault signals. Afterwards, the robust electromagnetic docking problem for spacecraft in the presence of multiple perturbations, e.g., external disturbances, fault signals, measurement errors, and input constraints, is investigated, and a novel intermediate observer was developed to estimate the relative motion information and the lumped disturbance [26]. It should be mentioned that the soft docking problem was not involved in [26], as no tracking trajectories were predefined during electromagnetic docking. Zhang et al. investigated a soft-docking controller in [27] with effective and satisfactory results, but the convergence time of system states is asymptotic, but not finite. Sliding mode control (SMC) is well known for its total insensitivity to both model uncertainties and external disturbances when the system states move on the sliding manifolds, which is the inherent strong robustness [28, 29]. In [30], the conventional linear sliding mode (LSM) control was used for underactuated electromagnetic docking in which each microsatellite was equipped with only one single coil, but the convergence of the system states to equilibrium point was also asymptotic. Meanwhile, as the system was underactuated, the attitudes of the two docking satellites were required to cooperate with each other to produce the desired intersatellite electromagnetic force. By designing a nonlinear sliding manifold, the terminal sliding mode (TSM) control was proposed and offers finite-time convergence and less steady-state errors [31]; however, there exist singularity problems. To avoid these singularity problems, a nonsingular terminal sliding mode (NTSM) controller which offers the advantages of LSM and TSM was presented for coupled spacecraft docking in [32]. In recent years, many controllers have been derived based on NTSM and have been used in many applications [33, 34]. However, there is only one linear term of the states in NTSM, which means NTSM controllers rapidly converge once the states approach the equilibrium and slowly converge once the states are far from the equilibrium.. To further increase the convergence rate of NTSM, the fastnonsingular terminal sliding mode (FNTSM) introduces both linear term and nonlinear term to design the sliding manifold, then the global fast convergence is guaranteed.

In this paper, a novel finite-time control scheme of electromagnetic docking is presented by combining the improved far-field model, a FNTSM controller, and a disturbance observer. The improved far-field model is proposed by introducing a multiplicative modification term into the farfield model, and the error of this improved model is restricted to a small and bounded range by the on-line self-adjusting of the modification term. Then, taking energy consumption as the index, the optimal direction of the magnetic moment is derived, and the calculation results indicate that such an optimal magnetic moment allocation can produce greater electromagnetic force with the existing input current amplitude. Furthermore, based on the improved far-field model and optimal magnetic moment allocation, a global fast nonsingular terminal sliding controller driven by an exponentially convergent disturbance observer for electromagnetic soft-docking is designed. It is chattering-free and possesses superior properties such as global fast finite-time convergence, no singularity, strong robustness, and highprecision control. Two scenarios are simulated to validate the effectiveness and merits of the developed control method. Finally, some conclusions are drawn. 


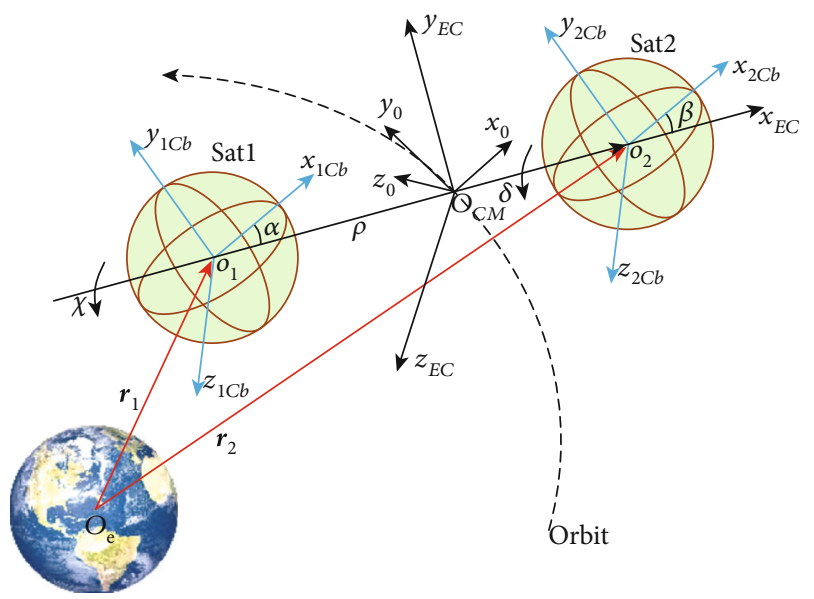

FIGURE 1: Coordinate systems and their relationships.

\section{Electromagnetic Docking Dynamics}

2.1. Coordinate Systems and Transformations. For convenience, the definitions of coordinate systems and their relationships are given first, as illustrated in Figure 1. The frame $O_{\mathrm{CM}} x_{O} y_{O} z_{O}$ is the orbital frame (abbreviated as the $\mathrm{CM}$ frame); its origin is located at the center of the mass of the spacecraft system (composed of Sat1 and Sat2). The frame $O_{\mathrm{CM}} x_{\mathrm{EC}} y_{\mathrm{EC}} z_{\mathrm{EC}}$ is the electromagnetic force/torque calculation frame (abbreviated as the EC frame); its origin is located at the center of mass of the spacecraft system, the $O_{\mathrm{CM}} x_{\mathrm{EC}}$ axis is aligned with the relative position vector from Sat1 to Sat2, the $O_{\mathrm{CM}} y_{\mathrm{EC}}$ axis is located at the orbital plane of the center of mass of the spacecraft system and defined by requirements, and the $O_{\mathrm{CM}} z_{\mathrm{EC}}$ axis completes the righthand rule. The frame $o_{1} x_{1 \mathrm{Cb}} y_{1 \mathrm{Cb}} z_{1 \mathrm{Cb}}$ is the body frame (abbreviated as the $\mathrm{Cb}$ frame); its origin is located at the center of the coil of Sat 1 , the $o_{1} x_{1 \mathrm{Cb}}$ axis is aligned with the axial direction of the coil, and $o_{1} y_{1 \mathrm{Cb}}$ axis and $o_{1} z_{1 \mathrm{Cb}}$ axis are located in the plane of the coil. The other two body frames of the coils of Sat1 can be obtained through their orthogonal relations. The body frames of the three orthogonal coils on Sat2 are the same as those on Sat1.

The transformation matrix from the CM frame to the EM frame is given by

$$
\begin{aligned}
& \boldsymbol{T}_{O}^{\mathrm{EC}}(\theta, \varphi)=\left[\begin{array}{ccc}
\cos \theta \cos \varphi & \cos \theta \sin \varphi & -\sin \theta \\
-\sin \varphi & \cos \varphi & 0
\end{array}\right. \\
& \left\{\begin{array}{l}
\theta=-\arcsin \left(\rho_{z} / \sqrt{\rho_{x}^{2}+\rho_{y}^{2}+\rho_{z}^{2}}\right), \\
\varphi=\arctan \left(\rho_{y} / \rho_{x}\right)
\end{array}\right. \\
& \boldsymbol{\rho}=\boldsymbol{r}_{2}-\boldsymbol{r}_{1}=\left[\rho_{x} \rho_{y} \rho_{z}\right]^{\mathrm{T}} .
\end{aligned}
$$

where $\boldsymbol{r}_{1}$ and $\boldsymbol{r}_{2}$ are the position vectors of Sat1 and Sat2, respectively, in the CM frame.
The transformation matrices from the $\mathrm{Cb}$ frames of Sat 1 and Sat 2 to the EC frame are given by

$$
\begin{aligned}
& \boldsymbol{T}_{1 \mathrm{Cb}}^{\mathrm{EC}}(\alpha, \chi)=\left[\begin{array}{ccc}
\cos \alpha & -\sin \alpha & 0 \\
\sin \alpha \cos \chi & \cos \alpha \cos \chi & -\sin \chi \\
\sin \alpha \sin \chi & \cos \alpha \sin \chi & \cos \chi
\end{array}\right], \\
& \boldsymbol{T}_{2 \mathrm{Cb}}^{\mathrm{EC}}(\beta, \delta)=\left[\begin{array}{ccc}
\cos \beta & -\sin \beta & 0 \\
\sin \beta \cos \delta & \cos \beta \cos \delta & -\sin \delta \\
\sin \beta \sin \delta & \cos \beta \sin \delta & \cos \delta
\end{array}\right],
\end{aligned}
$$

where $\alpha$ is the angle between the $o_{1} x_{1 \mathrm{Cb}}$ axis and the $O_{\mathrm{CM}} x_{\mathrm{EC}}$ axis; $\chi$ is the rotation angle of the $o_{1} x_{1 \mathrm{Cb}}$ axis about the $O_{\mathrm{CM}} x_{\mathrm{EC}}$ axis; $\beta$ and $\delta$ are similar to $\alpha$ and $\chi$, respectively.

2.2. Electromagnetic Force Models and Error Analysis. Take two current loops of carrying currents $i_{1}$ and $i_{2}$, respectively, as shown in Figure 2,

where $i_{1} \mathrm{~d} \boldsymbol{l}_{1}$ and $i_{2} \mathrm{~d} \boldsymbol{l}_{2}$ are the vectors of current elements; $\boldsymbol{a}_{1}$ and $\boldsymbol{a}_{2}$ are the radius vectors pointing to $i_{1} \mathrm{~d} \boldsymbol{l}_{1}$ and $i_{2} \mathrm{~d} \boldsymbol{l}_{2}$, respectively; $\boldsymbol{s}$ is the vector from the center of loop 1 to $i_{2} \mathrm{~d}$ $\boldsymbol{l}_{2} ; \boldsymbol{r}$ is the vector from $i_{1} \mathrm{~d} \boldsymbol{l}_{1}$ to $i_{2} \mathrm{~d} \boldsymbol{l}_{2}$.

According to the theory of electromagnetics, the electromagnetic force exerted on loop 2 by loop 1 is given by

$$
\boldsymbol{F}_{2}=\frac{\mu_{0} i_{1} i_{2}}{4 \pi} \oint\left(\oint \frac{\boldsymbol{r} \times \mathrm{d} \boldsymbol{l}_{1}}{|\boldsymbol{r}|^{3}}\right) \times \mathrm{d} \boldsymbol{l}_{2},
$$

where $\mu_{0}$ is the magnetic permeability of free space and is equal to $4 \pi \times 10^{-7} \mathrm{~T} \mathrm{~m} / \mathrm{A}$.

Eq. (4) is the exact model of electromagnetic force and cannot be solved analytically because of a double integration calculation with the magnitude of $\boldsymbol{r}^{3}$ in the denominator; thus, a numerical integrator must be employed.

At a sufficient distance from two coils, each coil can be represented by a magnetic dipole. Using the Taylor series expansion to expand $1 / \boldsymbol{r}$ at $\boldsymbol{r}=\boldsymbol{s}$, the far-field model [11] can be derived as

$$
\left\{\begin{array}{l}
\boldsymbol{F}_{2}=K\left(\frac{\boldsymbol{\mu}_{1} \times \boldsymbol{\mu}_{2}}{d^{5}} \boldsymbol{d}+\frac{\boldsymbol{\mu}_{1} \times \boldsymbol{d}}{d^{5}} \boldsymbol{\mu}_{2}+\frac{\boldsymbol{\mu}_{2} \times \boldsymbol{d}}{d^{5}} \boldsymbol{\mu}_{1}-5 \frac{\left(\boldsymbol{\mu}_{1} \times \boldsymbol{d}\right)\left(\boldsymbol{\mu}_{2} \times \boldsymbol{d}\right)}{d^{7}} \boldsymbol{d}\right) \\
\boldsymbol{\mu}_{i}=N_{i} i_{i} S_{i} \boldsymbol{n}_{i}, \quad(i=1,2), \\
K=\frac{3 \mu_{0}}{4 \pi}
\end{array}\right.
$$

where $\boldsymbol{\mu}_{1}$ and $\boldsymbol{\mu}_{2}$ are the magnetic moment vectors of the dipoles; $\boldsymbol{d}$ is the vector from the center of coil 1 to the center of coil 2; $N_{1}$ and $N_{2}$ are the numbers of turns of coil 1 and coil 2, respectively; $S_{1}$ and $S_{2}$ are the areas enclosed by the coils; $\boldsymbol{n}_{1}$ and $\boldsymbol{n}_{2}$ are the unit vectors along the axes of coil 1 and coil 2 , respectively; $K$ is a constant auxiliary parameter.

Equipping the coils on Sat1 and Sat2, two dipoles in the EC frame are shown in Figure 3. 


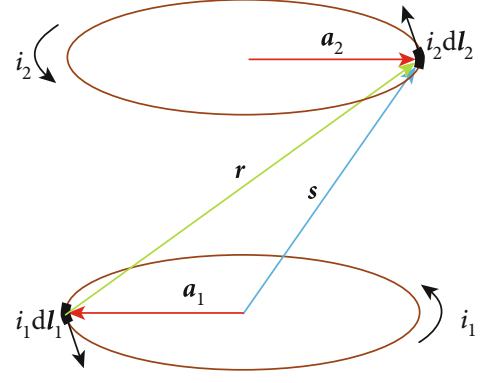

Figure 2: Two current loops.

The magnetic moment vectors can be given by

$$
\boldsymbol{\mu}_{i}=\left[\begin{array}{lll}
\mu_{i x} & \mu_{i y} & \mu_{i z}
\end{array}\right]^{\mathrm{T}}=\boldsymbol{T}_{i \mathrm{Cb}}^{\mathrm{EC}}\left[\begin{array}{ll}
\mu_{i} & 0
\end{array}\right]^{\mathrm{T}}, \quad(i=1,2) .
$$

Substituting Eq. (6) into Eq. (5) yields two representations of the far-field model

$$
\begin{aligned}
& \left\{\begin{array}{l}
F_{2 x}=K\left(-2 \mu_{1 x} \mu_{2 x}+\mu_{1 y} \mu_{2 y}+\mu_{1 z} \mu_{2 z}\right) / d^{4}, \\
F_{2 y}=K\left(\mu_{1 x} \mu_{2 y}+\mu_{1 y} \mu_{2 x}\right) / d^{4}, \\
F_{2 z}=K\left(\mu_{1 x} \mu_{2 z}+\mu_{1 z} \mu_{2 x}\right) / d^{4},
\end{array}\right. \\
& \left\{\begin{array}{l}
F_{2 x}=K \mu_{1} \mu_{2}(-2 \cos \alpha \cos \beta+\cos (\delta-\chi) \sin \alpha \sin \beta) / d^{4}, \\
F_{2 y}=K \mu_{1} \mu_{2}(\cos \alpha \sin \beta \cos \delta+\sin \alpha \cos \beta \cos \chi) / d^{4}, \\
F_{2 z}=K \mu_{1} \mu_{2}(\cos \alpha \sin \beta \sin \delta+\sin \alpha \cos \beta \sin \chi) / d^{4} .
\end{array}\right.
\end{aligned}
$$

Since the exact model is derived from electromagnetic theory, we regard its calculation results as the actual electromagnetic force. Define the percent error of the far-field model as

$$
e_{\text {far }}=\left(\left|\boldsymbol{F}^{\text {far }}\right|-\left|\boldsymbol{F}^{\text {exact }}\right|\right) /\left|\boldsymbol{F}^{\text {exact }}\right| \times 100 \%,
$$

where $\boldsymbol{F}^{\text {exact }}$ and $\boldsymbol{F}^{\text {far }}$ are the forces calculated by the exact model and the far-field model, respectively. Here, we use the double integral function "integral2" in MATLAB to calculate the exact electromagnetic force and compare its results with the calculation results of the far-field model.

When the axes of two coils are aligned $(\alpha=\beta=0)$, the force is only on the $O_{\mathrm{CM}} x_{\mathrm{EC}}$ axis, and as expected, the coils attract. Suppose the radii of the coils are $0.2 \mathrm{~m}$, the currents are $1 \mathrm{~A}$, the numbers of turns are 1000 , and the calculation results for the relative distance in the exact model and in the far-field model are shown in Figure 4.
In Figure 4, the left side shows that the forces calculated by the far-field model increase rapidly with the decrease in relative distance; in contrast, the forces calculated by the exact model increase slowly. However, the forces are approximatively equal at far distances. The right side shows the percent error of the far-field model. The percent error is $9 \%$ at $1.5 \mathrm{~m}$ and up to $4500 \%$ at $0.1 \mathrm{~m}$, and even infinity at $0 \mathrm{~m}$ (from Eq. (5) and Figure 4), which indicates hard docking or collision at the final phase of docking because of insufficient control. Thus, the relative distance has a strong influence on the error of the far-field model and cannot be ignored.

For the problem of model uncertainties, the general solution methods are robust control or disturbance observerbased control. Robust control, which is insensitive to model uncertainties, always comes at the expense of control accuracy and is not suitable for spacecraft docking because of the high-precision requirements. A disturbance observer that can estimate the model error and external disturbances usually has a lagging effect. When the relative distance decreases, the error of the far-field model increases rapidly, and thus, the tracking error of the disturbance observer will also increase (this is verified by simulations in Section 5). Thus, it is necessary to restrict the error of the far-field model to a slowly changing and bounded range, and then, the disturbance observer can be used more appropriately to estimate the model uncertainties to realize a high-precision model approach. In the next section, an improved far-field model is proposed by introducing a modification term to the farfield model.

2.3. Improved Far-Field Model. Eq. (9) may be written as

$$
\left|\boldsymbol{F}^{\mathrm{exact}}\right|=\left|\boldsymbol{F}^{\mathrm{far}}\right| /\left(1+e_{\mathrm{far}}\right)=\left|\boldsymbol{F}^{\mathrm{far}}\right| \times c,
$$

where $c$ denotes the modification term of the far-field model.

Generally, the radii of coils and the numbers of turns are determined, and the influence of currents on electromagnetic forces is linear; that is to say, the currents have no effect on the error of the far-field model. The relative orientations of the coils have complex effects on the error of the far-field model. Such effects are tightly coupled with the radii of the coils and the relative distance; however, compared with the effects of relative distance, they are small. Thus, in order to modify the far-field model, an approximation is adopted to solve the modification term $c$ about the relative distance parameter and make the term $c$ a multiplicative factor to add to the far-field model.

The rotational function approximation has the advantages of simple form, a small amount of computation, etc. Considering the trend of the error curve and the complexity of the modification term, the following rational fraction is selected to approximate the percent error of the far-field model

$$
e_{\mathrm{far}}(d)=1 /\left(p_{0} d^{3}+p_{1} d^{2}+p_{2} d+p_{3}\right)
$$




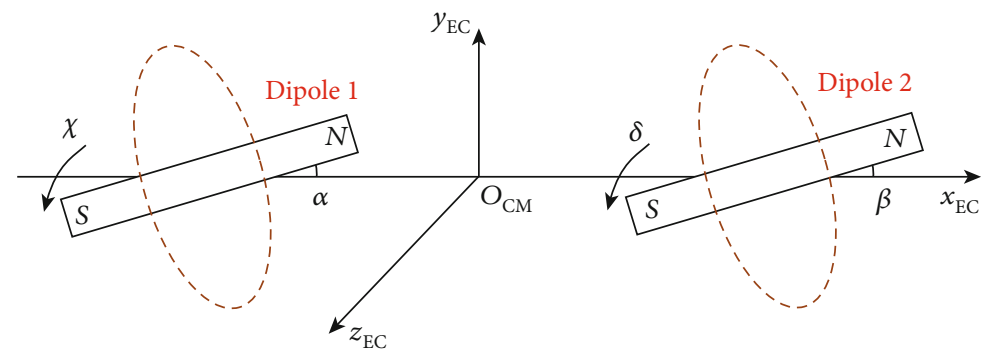

Figure 3: Relative position and attitude between two dipoles.
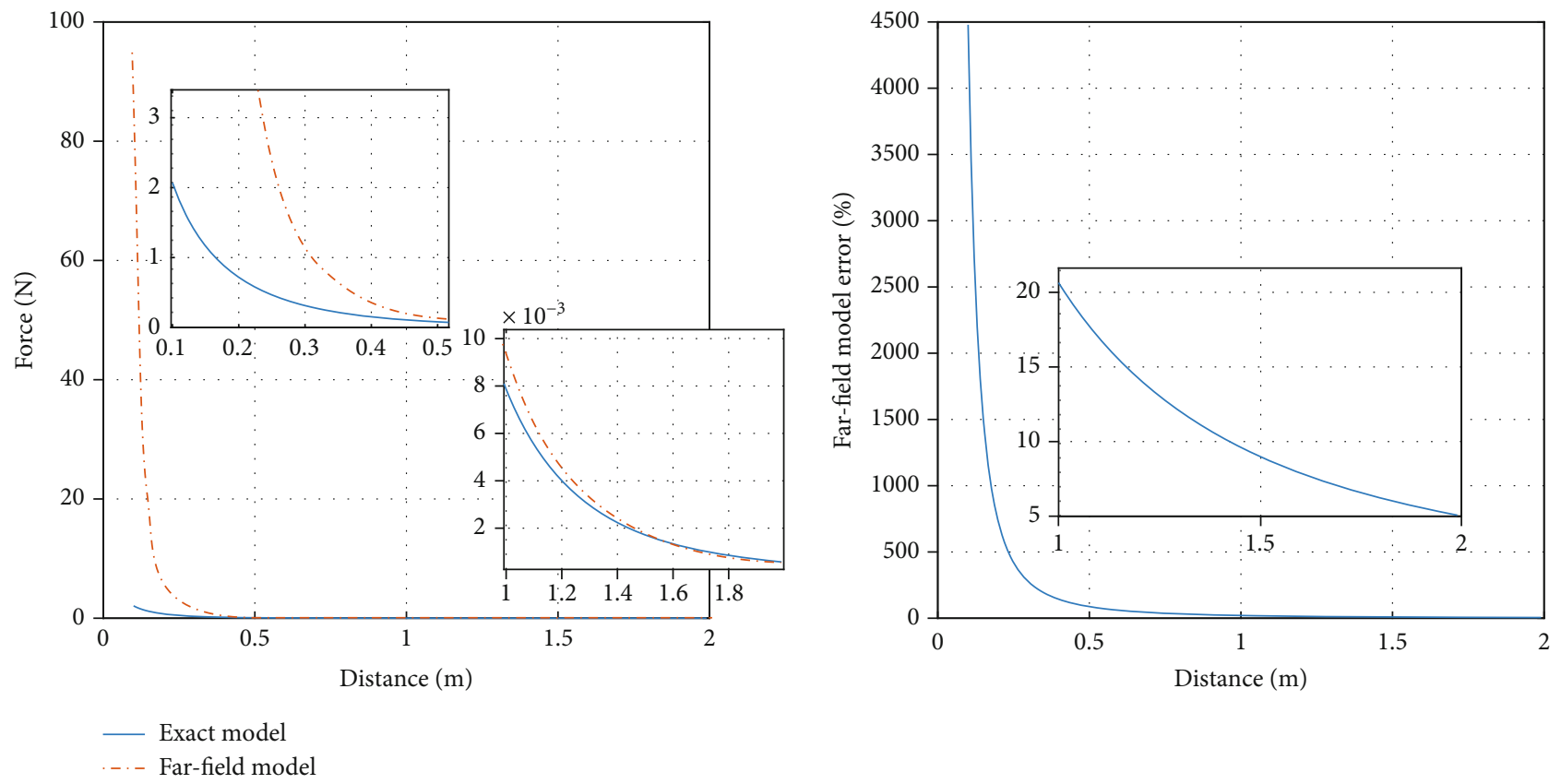

FIGURE 4: Calculation results and percent error of the far-field model.

There are four unknown parameters in Eq. (11), and four points on the error curve should be selected for interpolation. From Eq. (11), we have

$$
\left[\begin{array}{l}
p_{0} \\
p_{1} \\
p_{2} \\
p_{3}
\end{array}\right]=\left[\begin{array}{llll}
d_{1}^{3} & d_{1}^{2} & d_{1} & 1 \\
d_{2}^{3} & d_{2}^{2} & d_{2} & 1 \\
d_{3}^{3} & d_{3}^{2} & d_{3} & 1 \\
d_{4}^{3} & d_{4}^{2} & d_{4} & 1
\end{array}\right]^{-1}\left[\begin{array}{l}
1 / e_{\mathrm{far}}\left(d_{1}\right) \\
1 / e_{\mathrm{far}}\left(d_{2}\right) \\
1 / e_{\mathrm{far}}\left(d_{3}\right) \\
1 / e_{\mathrm{far}}\left(d_{4}\right)
\end{array}\right] .
$$

Taking the electromagnetic force characteristics into account, we take $d_{1}=0.05 \mathrm{~m}, d_{2}=0.08 \mathrm{~m}, d_{3}=0.15 \mathrm{~m}$, and $d_{4}=1.0 \mathrm{~m}$. Assuming that the axes of the two coils are aligned, the error term can be calculated by Eq. (12) as

$$
e_{\mathrm{far}}(d)=1 /\left(0.5407 d^{3}+4.6483 d^{2}-0.3155 d+0.0072\right)
$$

and the modification term can be presented as

$$
c(d)=1 /\left(1+e_{\mathrm{far}}(d)\right) .
$$

After modification, at the relative distance from $0.05 \mathrm{~m}$ to $2 \mathrm{~m}$, the calculation results and the percent error of the improved far-field model are shown in Figure 5.

As the figure shows, with the axes of the two coils aligned, modifying the far-field model by relative distance parameter only can reduce the percent error of the improved far-field model to within 5\%. When the relative attitude angle of the two coils is small, but not zero, the percent error of the improved far-field model is shown in Figure 6.

When the relative attitude angle of the two coils is less than $10^{\circ}$, if the relative distance is more than $0.5 \mathrm{~m}$, the percent error of the improved far-field model is within $5 \%$, and if the relative distance is more than $0.1 \mathrm{~m}$, the percent error is within $10 \%$. Generally, at the final phase of docking, the relative attitude of the two satellites has been controlled to a small angle, and in this case, the improved far-field model is available. 

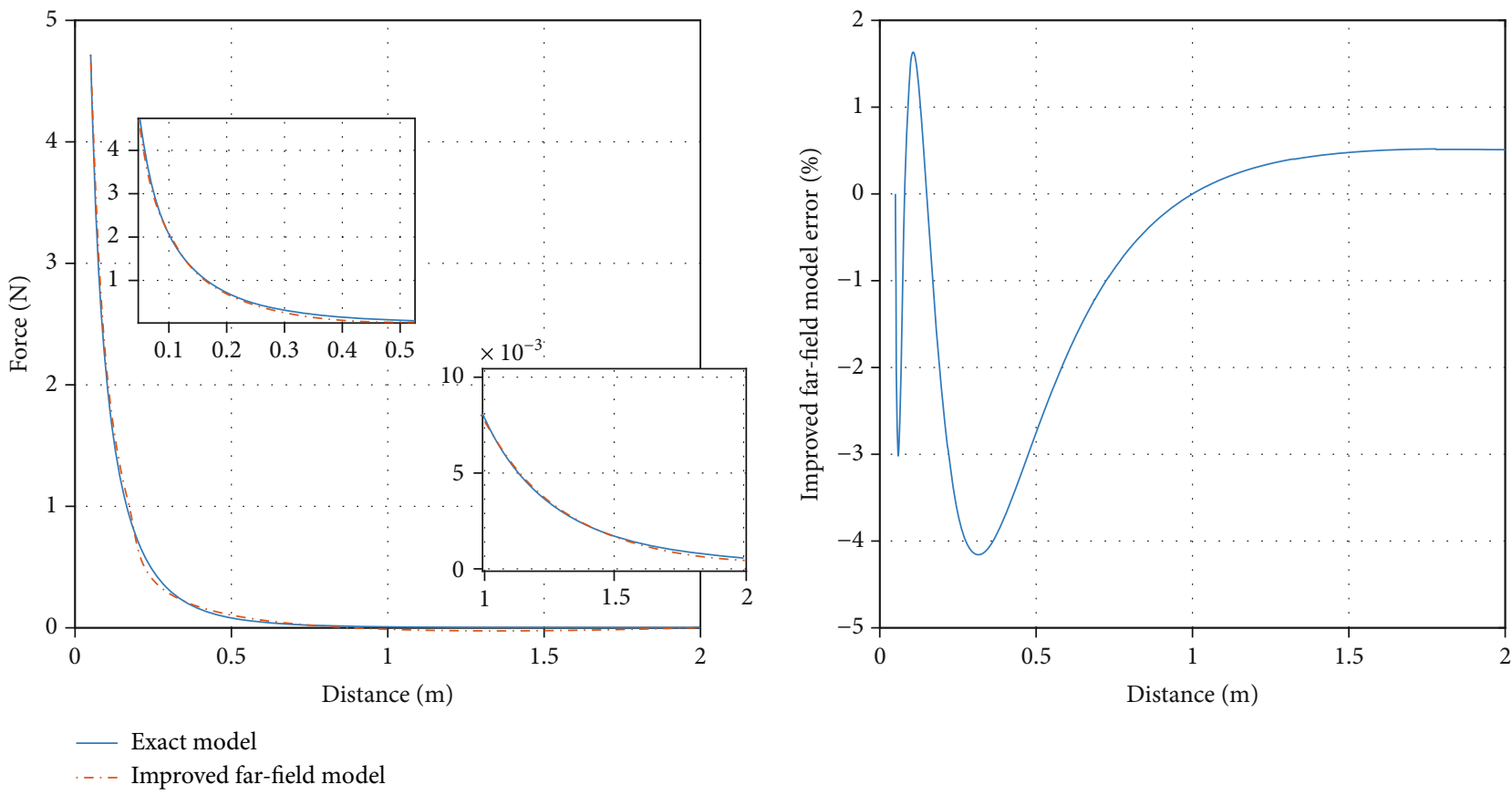

Figure 5: Calculation results and percent error of the improved far-field model.

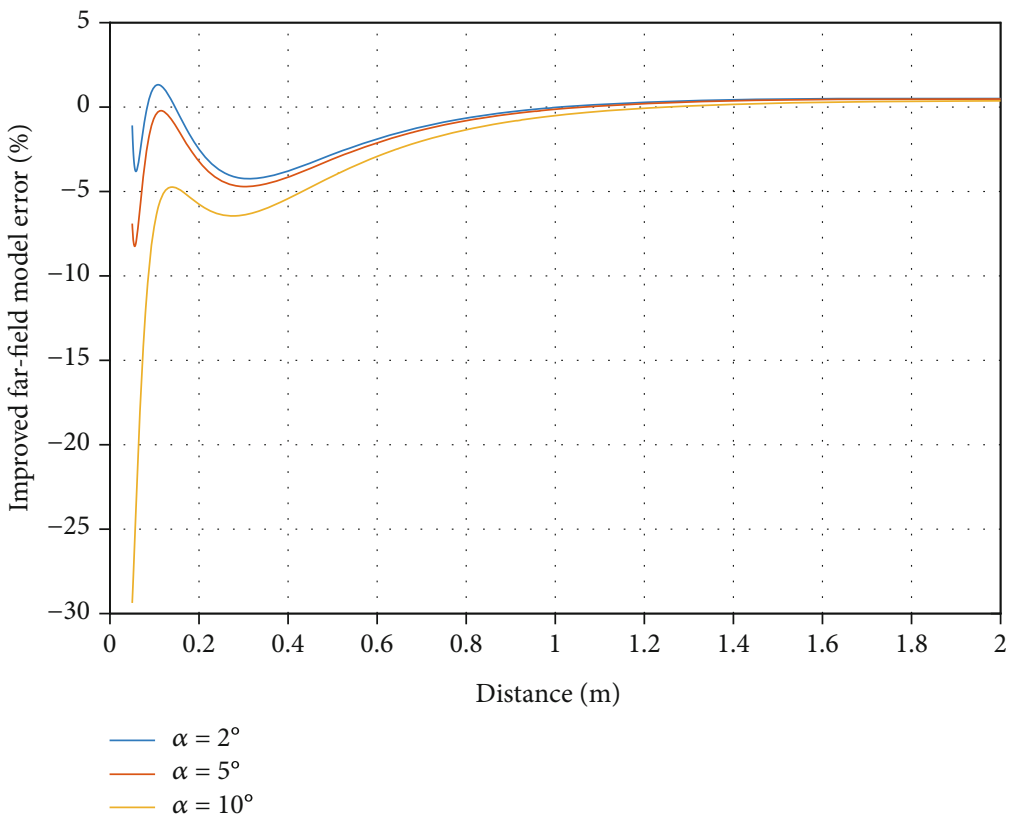

Figure 6: The percent error of the improved far-field model $(\alpha \neq 0)$.

It can be seen from the calculations and analysis above that compared with the far-field model, the model accuracy of the proposed improved far-field model is substantially higher. The error of the improved model, which can be regarded as a kind of unknown disturbance, is restricted to a limited and bounded range, and such error or disturbance can be estimated and compensated effectively by a disturbance observer. Moreover, in the improved far-field model, a modification term is introduced; this term is a function of relative distance, which implies that the far-field model can be corrected using the measurements of relative distance to realize on-line self-adjusting.

2.4. Translational Dynamics Model. Since the intersatellite electromagnetic forces change the motions of Sat1 and Sat2 simultaneously, the relative motion of Sat1 and Sat2 does not satisfy the $\mathrm{C}-\mathrm{W}$ equations. However, the intersatellite electromagnetic forces which are the internal forces of a 
spacecraft system have no impact on the motion of the center of mass of the spacecraft system; thus, the translational dynamics model can be established in the CM frame. It should be noted that spacecraft electromagnetic docking is a short-term operation at close range, so the relative motion has little influence on docking.

Assuming that the orbit of the center of mass of the spacecraft system is circular, the relative distance between Sat 1 and Sat2 is meter-sized. In the CM frame, the translational dynamics model of electromagnetic docking based on the improved far-field model with respect to the center of mass of the spacecraft system can be expressed as

$$
\left\{\begin{array}{l}
\ddot{\rho}_{x}-2 \omega \dot{\rho}_{y}-3 \omega^{2} \rho_{x}=\left(1 / m_{1}+1 / m_{2}\right)\left(c_{x}+\varepsilon_{x}\right) F_{2 x O}^{\mathrm{far}}+\Delta f_{x}, \\
\ddot{\rho}_{y}+2 \omega \dot{\rho}_{x}=\left(1 / m_{1}+1 / m_{2}\right)\left(c_{y}+\varepsilon_{y}\right) F_{2 y O}^{\mathrm{far}}+\Delta f_{y}, \\
\ddot{\rho}_{z}+\omega^{2} \rho_{z}=\left(1 / m_{1}+1 / m_{2}\right)\left(c_{z}+\varepsilon_{z}\right) F_{2 z O}^{\mathrm{far}}+\Delta f_{z},
\end{array}\right.
$$

where $\rho_{x}, \rho_{y}$, and $\rho_{z}$ are the relative position components; $\omega$ denotes the orbit angular velocity; $m_{1}$ and $m_{2}$ denote the masses of Sat1 and Sat2, respectively; $\Delta f_{x}, \Delta f_{y}$, and $\Delta f_{z}$ denote unknown disturbances; $c_{x}, c_{y}$, and $c_{z}$ denote the modification terms; $\varepsilon_{x}, \varepsilon_{y}$, and $\varepsilon_{z}$ denote the multiplicative error coefficients of the improved far-field model; and $F_{2 x O}^{\mathrm{far}}, F_{2 y O}^{\mathrm{far}}$, and $F_{2 z O}^{\mathrm{far}}$ denote the electromagnetic forces on Sat 2 calculated by the far-field model in the CM frame, whose relationships with actual electromagnetic forces $F_{2 i O}^{\text {exact }}$ can be expressed as

$$
F_{2 i O}^{\text {exact }}=\left(c_{i}+\varepsilon_{i}\right) F_{2 i O}^{\mathrm{far}}, \quad(i=x, y, z) .
$$

The dynamics model can be further simplified as

$$
\left\{\begin{array}{l}
\ddot{\rho}_{x}-2 \omega \dot{\rho}_{y}-3 \omega^{2} \rho_{x}=\left(1 / m_{1}+1 / m_{2}\right) c_{x} F_{2 x O}^{\mathrm{far}}+D_{x}, \\
\ddot{\rho}_{y}+2 \omega \dot{\rho}_{x}=\left(1 / m_{1}+1 / m_{2}\right) c_{y} F_{2 y O}^{\mathrm{far}}+D_{y} \\
\ddot{\rho}_{z}+\omega^{2} \rho_{z}=\left(1 / m_{1}+1 / m_{2}\right) c_{z} F_{2 z O}^{\mathrm{far}}+D_{z}
\end{array}\right.
$$

where $D_{x}, D_{y}$, and $D_{z}$ are the lumped disturbances consisting of the model uncertainties and unknown external disturbances and can be expressed as

$$
D_{i}=\left(1 / m_{1}+1 / m_{2}\right) \varepsilon_{i} F_{2 i O}^{\mathrm{far}}+\Delta f_{i}, \quad(i=x, y, z) .
$$

Since the electromagnetic forces are calculated in the EC frame and exerted on dynamics in the CM frame, the coordinate transformation should be applied as

$$
\boldsymbol{F}_{O}=\left[\begin{array}{lll}
F_{x O} & F_{y O} & F_{z O}
\end{array}\right]^{\mathrm{T}}=\boldsymbol{T}_{O}^{\mathrm{EC}}\left[\begin{array}{lll}
F_{x \mathrm{EC}} & F_{y \mathrm{EC}} & F_{z \mathrm{EC}}
\end{array}\right]^{\mathrm{T}}=\boldsymbol{T}_{O}^{\mathrm{EC}} \boldsymbol{F}_{\mathrm{EC}} .
$$

\section{Analysis of Three-Dimensional Magnetic Moment Allocation}

The directions of electromagnetic forces are determined as the relative position and orientation of two coils are fixed. Using EMFF as a reference, to obtain the forces and torques in arbitrary directions, each spacecraft is equipped with three orthogonal coils. Assuming that the attitude is controlled to the desired state by flywheels during the docking process, only the three components of the control force should be considered. That is, if Sat2 is equipped with three orthogonal coils, equipping Sat1 with a single coil is enough to produce arbitrary electromagnetic force. Thus, it is necessary to determine the optimal direction in which the axis of the single coil on Sat1 should point. The problem of determining the axis pointing direction of the coil is equivalent to the problem of how to distribute the magnetic moment produced by the coil. Thus, in this section, the magnetic moment allocation law is derived with the constraint of desired forces and the index of energy consumption.

According to the first expression in Eq. (7), the coefficient before $\mu_{1 x}$ is -2 , and we speculate that the magnetic moment on the $x$-axis has a greater contribution to electromagnetic forces. Thus, in the three-dimensional scenario, two cases will be discussed to analyze how to distribute the magnetic moments of Sat1 on three axes to minimize energy consumption. In the first case, the magnetic moments are distributed on the $x$-axis only; and in the second case, the magnetic moments are distributed on the $x$-axis, $y$-axis, and $z$-axis.

Case 1. The magnetic moments are distributed only on the $x$-axis $\left(\mu_{1 x 1} \neq 0, \mu_{1 y 1}=\mu_{1 z 1}=0\right)$.

In this case, the electromagnetic force formulas can be expressed as

$$
\left\{\begin{array}{l}
F_{x}=-2 K \mu_{1 x 1} \mu_{2 x 1} / d^{4}, \\
F_{y}=K \mu_{1 x 1} \mu_{2 y 1} / d^{4}, \\
F_{z}=K \mu_{1 x 1} \mu_{2 z 1} / d^{4} .
\end{array}\right.
$$

If the parameters of the coils on Sat 1 and Sat 2 are all the same, the energy consumption of the coils is equivalent to the magnitudes of the magnetic moments. Assuming that $F_{x}, F_{y}$, and $F_{z}$ are the components of the desired force, and the magnetic moment components of Sat 2 are derived as

$$
\left\{\begin{array}{l}
\mu_{2 x 1}=-d^{4} F_{x} /\left(2 K \mu_{1 x 1}\right), \\
\mu_{2 y 1}=d^{4} F_{y} /\left(K \mu_{1 x 1}\right) \\
\mu_{2 z 1}=d^{4} F_{z} /\left(K \mu_{1 x 1}\right)
\end{array}\right.
$$

Then, the sum of the squares of the components is

$$
Q_{1}=\mu_{2 x 1}^{2}+\mu_{2 y 1}^{2}+\mu_{2 z 1}^{2}=\frac{d^{8}}{K^{2}} \cdot \frac{\left(F_{x}^{2}+4 F_{y}^{2}+4 F_{z}^{2}\right)}{4 \mu_{1 x 1}^{2}}
$$


Case 2. The magnetic moments are distributed on the $x$-axis, $y$-axis, and $z$-axis.

In this case, the electromagnetic force formulas can be expressed as

$$
\left\{\begin{array}{l}
F_{x}=K\left(-2 \mu_{1 x 2} \mu_{2 x 2}+\mu_{1 y 2} \mu_{2 y 2}+\mu_{1 z 2} \mu_{2 z 2}\right) / d^{4} \\
F_{y}=K\left(\mu_{1 x 2} \mu_{2 y 2}+\mu_{1 y 2} \mu_{2 x 2}\right) / d^{4} \\
F_{z}=K\left(\mu_{1 x 2} \mu_{2 z 2}+\mu_{1 z 2} \mu_{2 x 2}\right) / d^{4} .
\end{array}\right.
$$

The magnetic moment components of Sat2 are derived as

$$
\left\{\begin{array}{l}
\mu_{2 x 2}=\frac{d^{4}\left(-\mu_{1 x 2}^{2} F_{x}+\mu_{1 x 2} \mu_{1 y 2} F_{y}+\mu_{1 x 2} \mu_{1 z 2} F_{z}\right)}{K\left(2 \mu_{1 x 2}^{3}+\mu_{1 x 2} \mu_{1 y 2}^{2}+\mu_{1 x 2} \mu_{1 z 2}^{2}\right)} \\
\mu_{2 y 2}=\frac{d^{4}\left[\mu_{1 x 2} \mu_{1 y 2} F_{x}+\left(2 \mu_{1 x 2}^{2}+\mu_{1 z 2}^{2}\right) F_{y}-\mu_{1 y 2} \mu_{1 z 2} F_{z}\right]}{K\left(2 \mu_{1 x 2}^{3}+\mu_{1 x 2} \mu_{1 y 2}^{2}+\mu_{1 x 2} \mu_{1 z 2}^{2}\right)} \\
\mu_{2 z 2}=\frac{d^{4}\left[\mu_{1 x 2} \mu_{1 z 2} F_{x}-\mu_{1 y 2} \mu_{1 z 2} F_{y}+\left(2 \mu_{1 x 2}^{2}+\mu_{1 y 2}^{2}\right) F_{z}\right]}{K\left(2 \mu_{1 x 2}^{3}+\mu_{1 x 2} \mu_{1 y 2}^{2}+\mu_{1 x 2} \mu_{1 z 2}^{2}\right)}
\end{array}\right.
$$

Then, the sum of the squares of the components is

$$
Q_{2}=\mu_{2 x 2}^{2}+\mu_{2 y 2}^{2}+\mu_{2 z 2}^{2}=\frac{d^{8}}{K^{2}} \cdot \frac{n}{\mu_{1 x 2}^{2}\left(2 \mu_{1 x 2}^{2}+\mu_{1 y 2}^{2}+\mu_{1 z 2}^{2}\right)^{2}}
$$

where $n$ is derived as

$$
\begin{aligned}
n= & \left(-\mu_{1 x 2}^{2} F_{x}+\mu_{1 x 2} \mu_{1 y 2} F_{y}+\mu_{1 x 2} \mu_{1 z 2} F_{z}\right)^{2} \\
& +\left[\mu_{1 x 2} \mu_{1 y 2} F_{x}+\left(2 \mu_{1 x 2}^{2}+\mu_{1 z 2}^{2}\right) F_{y}-\mu_{1 y 2} \mu_{1 z 2} F_{z}\right]^{2} \\
& +\left[\mu_{1 x 2} \mu_{1 z 2} F_{x}-\mu_{1 y 2} \mu_{1 z 2} F_{y}+\left(2 \mu_{1 x 2}^{2}+\mu_{1 y 2}^{2}\right) F_{z}\right]^{2} .
\end{aligned}
$$

Dividing Eq. (25) by Eq. (22) yields

$$
\frac{Q_{2}}{Q_{1}}=1+\frac{m}{\left[F_{x}^{2}+4\left(F_{y}^{2}+F_{z}^{2}\right)\right] \mu_{1 x 2}^{2}\left(2 \mu_{1 x 2}^{2}+\mu_{1 y 2}^{2}+\mu_{1 z 2}^{2}\right)^{2}},
$$

where $m$ is defined as

$$
m=4 \mu_{1 x 1}^{2} n\left[F_{x}^{2}+4\left(F_{y}^{2}+F_{z}^{2}\right)\right] \mu_{1 x 2}^{2}\left(2 \mu_{1 x 2}^{2}+\mu_{1 y 2}^{2}+\mu_{1 z 2}^{2}\right)^{2} .
$$

Therefore, we can compare the sizes of $Q_{2}$ and $Q_{1}$ by the sign of $m$. When the magnetic moment components in
Case 2 are all distributed to the $x$-axis, as in Case 1 , then $\left|\mu_{1 x 1}\right|=\left|\mu_{1 x 2}\right|+\left|\mu_{1 y 2}\right|+\left|\mu_{1 z 2}\right|$, thus,

$$
\begin{aligned}
\mu_{1 x 1}^{2}= & \mu_{1 x 2}^{2}+\mu_{1 y 2}^{2}+\mu_{1 z 2}^{2}+2\left|\mu_{1 x 2}\right|\left|\mu_{1 y 2}\right| \\
& +2\left|\mu_{1 x 2}\right|\left|\mu_{1 z 2}\right|+2\left|\mu_{1 y 2}\right|\left|\mu_{1 z 2}\right| .
\end{aligned}
$$

Substituting Eq. (26) and Eq. (29) into Eq. (28) and making the terms whose signs are uncertain strictly negative, then based on mean value inequality, $m$ is derived as

$$
\begin{aligned}
m \geq & F_{x}^{2} \mu_{1 x 2}^{2}\left(3 \mu_{1 y 2}^{4}+6 \mu_{1 y 2}^{2} \mu_{1 z 2}^{2}+3 \mu_{1 z 2}^{4}+4 \mu_{1 y 2}^{2}\left|\mu_{1 x 2} \mu_{1 y 2}\right|\right. \\
& \left.+4 \mu_{1 z 2}^{2}\left|\mu_{1 x 2} \mu_{1 z 2}\right|\right)+F_{y}^{2} \mu_{1 x 2}^{2}\left[4 \mu_{1 x 2}^{2} \mu_{1 z 2}^{2}+\left|\mu_{1 x 2} \mu_{1 y 2}\right|\right. \\
& \left.\cdot\left(28 \mu_{1 x 2}^{2}+4 \mu_{1 z 2}^{2}\right)+\left|\mu_{1 x 2} \mu_{1 z 2}\right|\left(28 \mu_{1 x 2}^{2}+8 \mu_{1 y 2}^{2}+8 \mu_{1 z 2}^{2}\right)\right] \\
& +F_{z}^{2} \mu_{1 x 2}^{2}\left[4 \mu_{1 x 2}^{2} \mu_{1 y 2}^{2}+\left|\mu_{1 x 2} \mu_{1 z 2}\right|\left(28 \mu_{1 x 2}^{2}+4 \mu_{1 y 2}^{2}\right)\right. \\
& \left.+\left|\mu_{1 x 2} \mu_{1 y 2}\right|\left(28 \mu_{1 x 2}^{2}+8 \mu_{1 z 2}^{2}+8 \mu_{1 y 2}^{2}\right)\right] \\
& +\left(F_{y}^{2} \mu_{1 x 2}^{2}+F_{z}^{2} \mu_{1 x 2}^{2}\right)\left|\mu_{1 y 2} \mu_{1 z 2}\right|\left(20 \mu_{1 x 2}^{2}+8 \mu_{1 z 2}^{2}+8 \mu_{1 y 2}^{2}\right)>0
\end{aligned}
$$

According to Eq. (30), if $\mu_{1 y 2}=\mu_{1 y 2}=0$, then $m=0$ results in $Q_{2}=Q_{1}$, which means the energy consumption of Sat1 in Case 1 is equal to its energy consumption in Case 2. If $\mu_{1 y 2}=0$ or $\mu_{1 z 2}=0$, then $m$ has a similar form, which can be determined from the expressions of the far-field model on the $y$-axis and the $z$-axis. Thus, if the pointing direction of the magnetic moment of Sat2 is not along the $x$-axis, then $m>0$, which means

$$
Q_{2} / Q_{1}>1
$$

In conclusion, when the desired forces are satisfied, if the magnetic moment of Sat1 points to Sat2, the energy consumption of Sat 2 will be minimized. Thus, the numerical simulations in Section 5 will follow this law.

To further validate the derivation results above for different desired forces, the energy consumption of Sat 2 is calculated at different magnetic moment allocations of Sat1. First, note that the expressions of the far-field model on the $y$-axis and the $z$-axis are the same. Then, according to the definition of the EC frame, the $y$-axis and the $z$-axis do not have unique definitions and may be defined differently according to the actual situation. Therefore, arbitrary desired forces can be described in the EC frame by the components of the $x$-axis and the $y$-axis or the $x$-axis and the $z$-axis; that is to say, it is reasonable to demonstrate the derivation results in two dimensions.

Assume that the magnitude of the magnetic moment of Sat 1 is a constant equal to $500 \pi \mathrm{A} \cdot \mathrm{m}^{2}$ that is distributed on the $x$-axis and the $y$-axis. The following figures show the energy consumption of Sat 2 relative to the different magnetic moment allocations of Sat 1 at four different desired forces. 

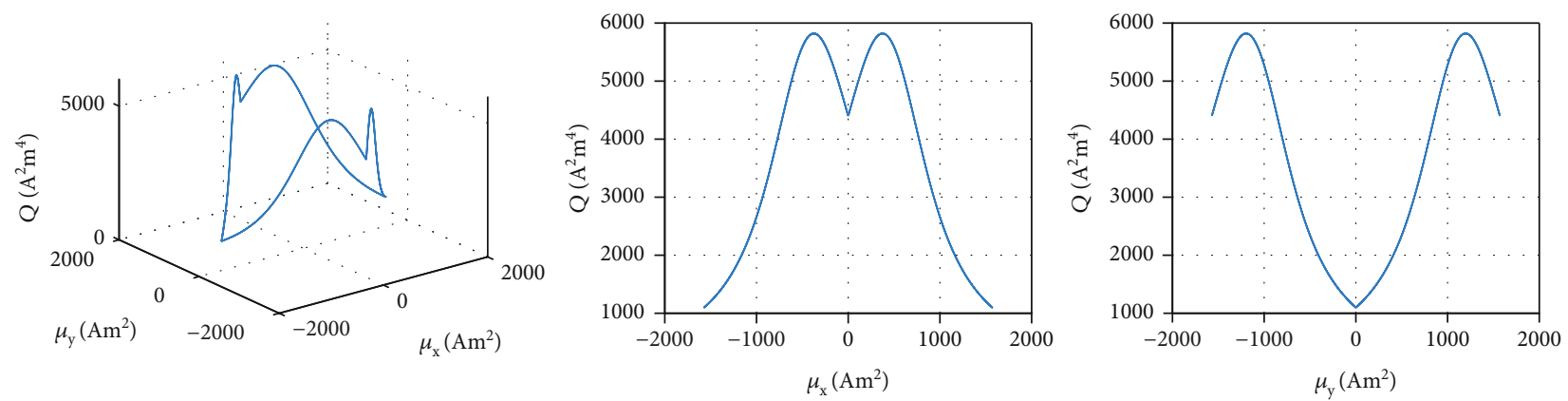

FIgure 7: The desired force is $[0.5 \mathrm{~N}, 0,0]^{\mathrm{T}}$.
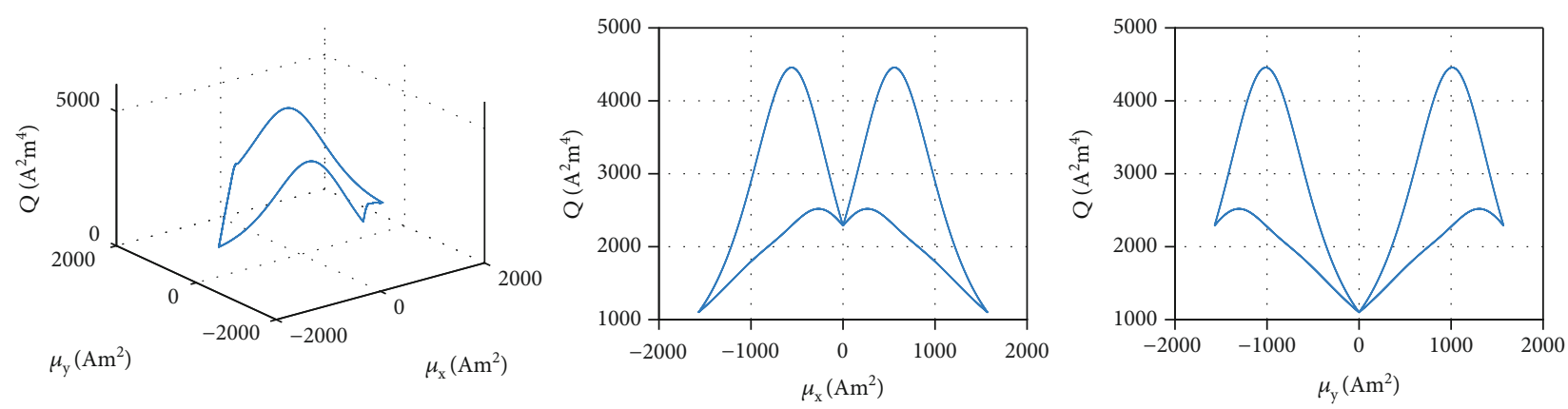

Figure 8: The desired force is $[0.3 \mathrm{~N}, 0.2 \mathrm{~N}, 0]^{\mathrm{T}}$.
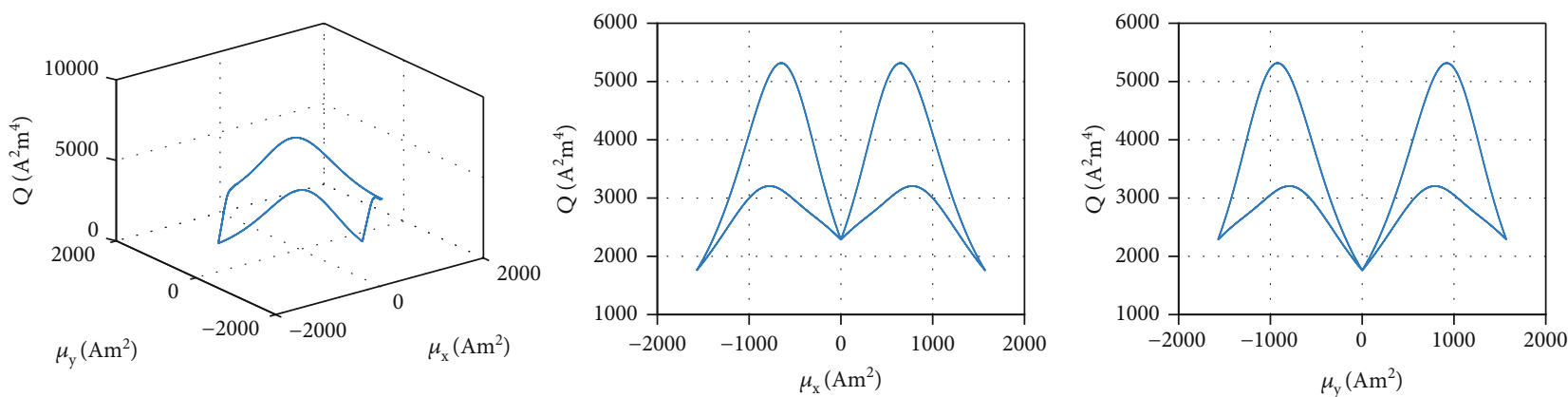

FIgure 9: The desired force is $[0.2 \mathrm{~N}, 0.3 \mathrm{~N}, 0]^{\mathrm{T}}$.
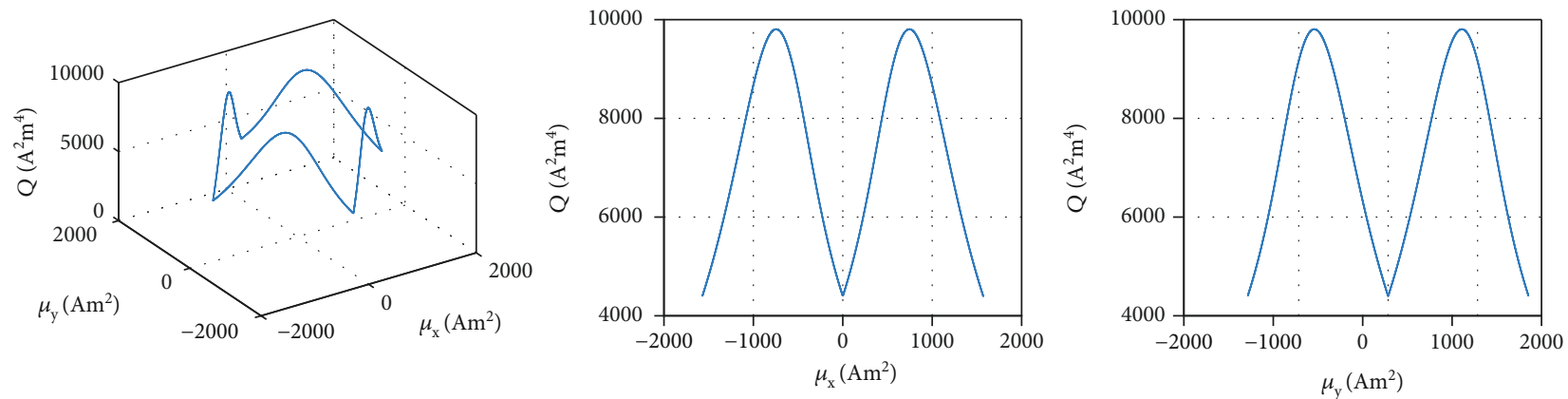

Figure 10: The desired force is $[0,0.5 \mathrm{~N}, 0]^{\mathrm{T}}$.

The figures on the left are the three-dimensional calculation results, and the figures in the middle and on the right are the elevation views and side views, respectively.
Figure 7 shows the calculation results when the desired force is on the $x$-axis. When the magnetic moments of Sat1 are all distributed to the $x$-axis, the value $Q$ is lowest, 


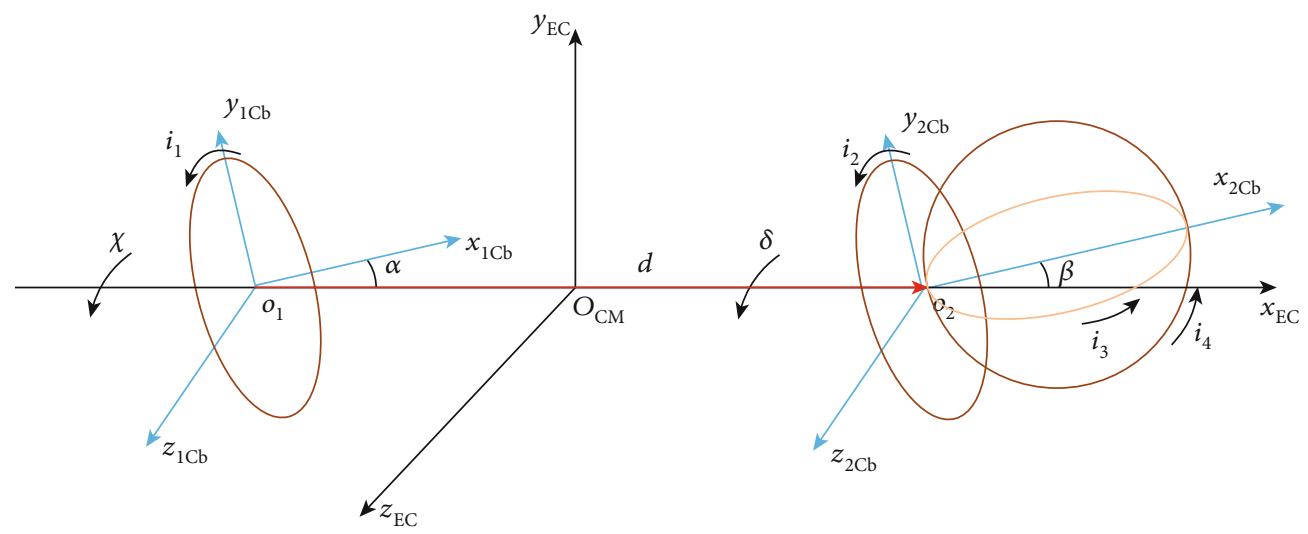

Figure 11: Configurations of the coils on Sat1 and Sat2 in the EC frame.

which means that the energy consumption of Sat1 is lowest. The greatest value $Q$ is produced not when the magnetic moments of Sat1 are all distributed to the $y$-axis but rather when the magnetic moments are distributed to both the $x$-axis and the $y$-axis. The conclusions shown in Figures 8 and 9 are the same as in Figure 7. However, as shown in Figure 10, when the desired force is on the $y$-axis and the magnetic moments of Sat1 are distributed to the $x$-axis or the $y$-axis, the value of $Q$ is the lowest. Thus, if the magnetic moment of Sat1 points to Sat2, it can be guaranteed that the energy consumption of Sat2 is the lowest for arbitrary desired forces.

According to the derivation and the verification results above, two conclusions can be drawn: (1) when the magnetic moments of Sat 1 and Sat 2 satisfy the desired forces, the direction in which the magnetic moment of Sat1 points impacts the energy consumption of Sat2, and in particular, the lowest energy consumption of Sat2 occurs when the magnetic moment of Sat1 points to Sat2; (2) from another point of view, when the magnetic moment of Sat1 points to Sat2, a larger force can be produced when the constraints of the currents of Sat 2 are considered.

\section{Electromagnetic Docking Control Scheme}

4.1. Control Objective. According to the derivation of magnetic moment allocation, assume Sat1 is equipped with a single coil along the docking axis and Sat 2 is equipped with three orthogonal coils. Generally, in EMFF, three orthogonal coils are configured with their centroids coinciding. However, in electromagnetic docking, there are greater electromagnetic forces when the dominant docking coil is configured in the form of offset along the docking axis. Here, we assume that the magnitude of the offset along the docking axis is equal to the radius of the coil; thus, the coils of Sat 1 and Sat 2 are configured as shown in the Figure 11.

As the figure shows, the left coil is coil 1, and the right coils are coil 2, coil 3, and coil 4, respectively. Assume that the magnetic moment of Sat1 is a constant that points to Sat 2 and that the magnitudes and directions of the electromagnetic forces are controlled by adjusting the currents on each coil of Sat2.
The transformation matrices from the $\mathrm{Cb}$ frames of coil 1 and coil 2 to the EC frame are given in Eq. (2) and Eq. (3), respectively. According to the orthogonal relation, the transformation matrices from the $\mathrm{Cb}$ frames of coil 3 and coil 4 to the EC frame are derived as

$$
\begin{aligned}
& \boldsymbol{T}_{3 \mathrm{Cb}}^{\mathrm{EC}}=\boldsymbol{T}_{2 \mathrm{Cb}}^{\mathrm{EC}} \boldsymbol{M}_{z}(-\pi / 2), \\
& \boldsymbol{T}_{4 \mathrm{Cb}}^{\mathrm{EC}}=\boldsymbol{T}_{2 \mathrm{Cb}}^{\mathrm{EC}} \boldsymbol{M}_{y}(\pi / 2) .
\end{aligned}
$$

where $\boldsymbol{M}_{y}(\cdot)$ and $\boldsymbol{M}_{z}(\cdot)$ are the unit transformation matrices.

The magnetic moment vectors produced by coil 1, coil 2, coil 3, and coil 4 at their $\mathrm{Cb}$ frames are expressed as

$$
\boldsymbol{\mu}_{i \mathrm{Cb}}=\left[\begin{array}{lll}
\mu_{i} & 0 & 0
\end{array}\right]^{\mathrm{T}}, \quad(i=1,2,3,4)
$$

By controlling the independent currents of the orthogonal coils on Sat2, a composed magnetic moment can be generated. Thus, in the EC frame, the magnetic moment vectors of Sat 1 and Sat 2 can be expressed as

$$
\begin{aligned}
& \boldsymbol{\mu}_{1}=\boldsymbol{T}_{1 \mathrm{Cb}}^{\mathrm{EC}} \boldsymbol{\mu}_{1 \mathrm{Cb}}, \\
& \boldsymbol{\mu}_{2}=\boldsymbol{T}_{2 \mathrm{Cb}}^{\mathrm{EC}} \boldsymbol{\mu}_{2 \mathrm{Cb}}+\boldsymbol{T}_{3 \mathrm{Cb}}^{\mathrm{EC}} \boldsymbol{\mu}_{3 \mathrm{Cb}}+\boldsymbol{T}_{4 \mathrm{Cb}}^{\mathrm{EC}} \boldsymbol{\mu}_{4 \mathrm{Cb}} .
\end{aligned}
$$

Substituting these two expressions into the improved far-field model yields the electromagnetic force on Sat2:

$$
\left[\begin{array}{l}
F_{2 x \mathrm{EC}} \\
F_{2 y \mathrm{EC}} \\
F_{2 z \mathrm{EC}}
\end{array}\right]=\frac{3 \mu_{0} \mu_{1}}{4 \pi} \boldsymbol{A} \boldsymbol{C} \boldsymbol{\mu}_{2}=\frac{3 \mu_{0} \mu_{1}}{4 \pi} \boldsymbol{A}\left[\begin{array}{ccc}
c_{11} & c_{12} & c_{13} \\
c_{21} & c_{22} & c_{23} \\
c_{31} & c_{32} & c_{33}
\end{array}\right]\left[\begin{array}{l}
\mu_{2} \\
\mu_{3} \\
\mu_{4}
\end{array}\right],
$$

where

$$
\boldsymbol{A}=\left[\begin{array}{ccc}
c_{x} d^{-4} & 0 & 0 \\
0 & c_{y}(d+a)^{-4} & 0 \\
0 & 0 & c_{z}(d+a)^{-4}
\end{array}\right]
$$




$$
\left\{\begin{array}{l}
c_{11}=-2 \cos \alpha \cos \beta+\sin \alpha \sin \beta \cos (\delta-\chi), \\
c_{12}=2 \cos \alpha \sin \beta+\sin \alpha \cos \beta \cos (\delta-\chi), \\
c_{13}=-\sin \alpha \sin (\delta-\chi), \\
c_{21}=\cos \alpha \sin \beta \cos \delta+\sin \alpha \cos \chi \cos \beta, \\
c_{22}=\cos \alpha \cos \beta \cos \delta-\sin \alpha \cos \chi \sin \beta, \\
c_{23}=-\cos \alpha \sin \delta \\
c_{31}=\cos \alpha \sin \beta \sin \delta+\sin \alpha \sin \chi \cos \beta, \\
c_{32}=\cos \alpha \cos \beta \sin \delta-\sin \alpha \sin \chi \sin \beta, \\
c_{33}=\cos \alpha \cos \delta
\end{array}\right.
$$

In Eq. (35), coil radius $a \neq 0, c_{x}, c_{y}$ are $c_{z}$ are the modification terms of the improved far-field model and not equal to zero; during docking, relative distance $d \neq 0$ and $\mu_{1} \neq 0$, so $\operatorname{det} \boldsymbol{A} \neq 0$; moreover, it can be calculated that $\operatorname{det} C=-2 \neq 0$ when the magnetic moment vector of Sat 1 points to Sat2. Thus, according to the desired electromagnetic force, the desired magnetic moment vector of Sat2 can be solved as

$$
\boldsymbol{\mu}_{2}=\left[\begin{array}{l}
\mu_{2} \\
\mu_{3} \\
\mu_{4}
\end{array}\right]=\frac{4 \pi}{3 \mu_{0} \mu_{1}} \boldsymbol{C}^{-1} \boldsymbol{A}^{-1}\left[\begin{array}{c}
F_{2 x \mathrm{EC}} \\
F_{2 y \mathrm{EC}} \\
F_{2 z \mathrm{EC}}
\end{array}\right] .
$$

The translational dynamics model of electromagnetic docking can be rewritten as

$$
\ddot{\rho}=f(\rho, \dot{\rho})+g(\rho) U+D,
$$

where

$$
\begin{aligned}
d & =\left(\rho_{x}^{2}+\rho_{y}^{2}+\rho_{z}^{2}\right)^{1 / 2}, \\
\boldsymbol{f}(\boldsymbol{\rho}, \dot{\boldsymbol{\rho}}) & =\left[\begin{array}{lll}
2 \omega \dot{\rho}_{y}+3 \omega^{2} \rho_{x} & -2 \omega \dot{\rho}_{x} & -\omega^{2} \rho_{z}
\end{array}\right]^{\mathrm{T}}, \\
\boldsymbol{g}(\boldsymbol{\rho}) & =\frac{3 \mu_{0} \mu_{1}}{4 \pi}\left(1 / m_{1}+1 / m_{2}\right) \boldsymbol{T}_{O}^{\mathrm{EC}} C, \\
\boldsymbol{U} & =\left[\begin{array}{lll}
\mu_{2} & \mu_{3} & \mu_{4}
\end{array}\right]^{\mathrm{T}}, \\
\boldsymbol{D} & =\left[\begin{array}{lll}
\boldsymbol{D}_{x} & \boldsymbol{D}_{y} & \boldsymbol{D}_{z}
\end{array}\right]^{\mathrm{T}} .
\end{aligned}
$$

It should be noted that the actual control variables are the currents on the coils of Sat2. Since the turns and radii of the coils are fixed, the magnetic moments are equivalent to the currents. Thus, the magnetic moment components of Sat 2 are chosen as the control variables.

According to the dynamics model described by Eq. (39), the control objectives are expressed as

$$
\boldsymbol{\rho}(t) \longrightarrow \boldsymbol{\rho}_{d}(t), \dot{\boldsymbol{\rho}}(t) \longrightarrow \dot{\boldsymbol{\rho}}_{d}(t),
$$

where $\boldsymbol{\rho}_{d}(t)$ and $\dot{\boldsymbol{\rho}}_{d}(t)$ are the desired relative position and velocity vectors, respectively.
The tracking errors are defined as

$$
\boldsymbol{e}=\boldsymbol{\rho}(t)-\boldsymbol{\rho}_{d}(t), \quad \dot{e}=\dot{\boldsymbol{\rho}}(t)-\dot{\boldsymbol{\rho}}_{d}(t)
$$

The goal of spacecraft electromagnetic docking control is to design a control scheme that guarantees that tracking errors converge to zero in finite time and that is robust to model uncertainties and external disturbances.

4.2. Disturbance Observer Design. Since model uncertainties and unknown external disturbances have an adverse impact on docking control performance, robustness and highprecision control are required. Using a disturbance observer, disturbance information can be captured to compensate for the dynamics model, so that the control system is guaranteed to have an anti-interference capability. Furthermore, to realize docking perfectly, fast convergence and high-precision tracking are crucial for the disturbance observer. Thus, based on dynamics characteristics, a disturbance observer with an exponential convergence rate is designed for electromagnetic docking.

Eq. (39) can be rewritten as

$$
\boldsymbol{D}=\ddot{\boldsymbol{\rho}}-f(\boldsymbol{\rho}, \dot{\boldsymbol{\rho}})-g(\boldsymbol{\rho}) U
$$

The main idea of the disturbance observer design is to modify the disturbance estimates by the differences between estimated outputs and actual outputs. Thus, the disturbance observer is designed as

$$
\begin{aligned}
& \dot{\hat{\mathbf{D}}}=\boldsymbol{K} \tilde{\boldsymbol{D}}=\boldsymbol{K}(\boldsymbol{D}-\widehat{\boldsymbol{D}})=-\boldsymbol{K} \widehat{\boldsymbol{D}}+\boldsymbol{K}(\ddot{\boldsymbol{\rho}}-\boldsymbol{f}(\boldsymbol{\rho}, \dot{\boldsymbol{\rho}})-\boldsymbol{g}(\boldsymbol{\rho}) \boldsymbol{U}), \\
& \boldsymbol{K}=\operatorname{diag}\left(K_{x}, K_{y}, K_{z}\right),
\end{aligned}
$$

where $\widehat{D}$ denotes the disturbance estimates; $K_{x}, K_{y}$, and $K_{z}$ are positive constants; $\tilde{\boldsymbol{D}}$ denotes the disturbance estimate error.

To avoid using high-order differential information of state variables, an auxiliary variable is defined as

$$
V=\widehat{D}-K \dot{\rho}
$$

Thus,

$$
\dot{V}=\dot{\widehat{D}}-K \ddot{\rho}=-K \widehat{D}+K(-f(\rho, \dot{\rho})-g(\rho) U)
$$

Then, the disturbance observer is rewritten as

$$
\left\{\begin{array}{l}
\dot{V}=-K \widehat{D}+K(-f(\rho, \dot{\rho})-g(\rho) U) \\
\widehat{D}=V+K \dot{\rho}
\end{array}\right.
$$

For constant disturbance or slowly time-varying disturbance, assuming $\dot{D} \doteq 0$ yields

$$
\dot{\tilde{D}}=\dot{D}-\dot{\widehat{D}} \doteq-\dot{\widehat{D}}=-\dot{V}-K \ddot{\rho} .
$$


Further derivation yields

$$
\begin{aligned}
\dot{\tilde{D}} & =-\dot{\boldsymbol{V}}-\boldsymbol{K} \ddot{\boldsymbol{\rho}}=-(-\boldsymbol{K} \widehat{\boldsymbol{D}}+\boldsymbol{K}(-\boldsymbol{f}(\boldsymbol{\rho}, \dot{\boldsymbol{\rho}})-\boldsymbol{g}(\boldsymbol{\rho}) \boldsymbol{U}))-\boldsymbol{K} \ddot{\boldsymbol{\rho}} \\
& =\boldsymbol{K}(-(\ddot{\boldsymbol{\rho}}-\boldsymbol{f}(\boldsymbol{\rho}, \dot{\boldsymbol{\rho}})-\boldsymbol{g}(\boldsymbol{\rho}) \boldsymbol{U})+\widehat{D})=\boldsymbol{K}(-\boldsymbol{D}+\widehat{D})=-\boldsymbol{K} \tilde{D}
\end{aligned}
$$

Thus,

$$
\dot{\tilde{D}}+K \tilde{D}=0
$$

The solution of this equation is

$$
\tilde{\boldsymbol{D}}(t)=\boldsymbol{K}_{\mathrm{e}} \tilde{\boldsymbol{D}}(0)=\operatorname{diag}\left(\mathrm{e}^{-K_{x} t}, \mathrm{e}^{-K_{y} t}, \mathrm{e}^{-K_{z} t}\right) \tilde{\boldsymbol{D}}(0),
$$

where $K_{\mathrm{e}}$ is a diagonal matrix. Because $\tilde{D}(0)$ is a constant vector determined by the initial time, the designed disturbance observer converges exponentially, and the convergence accuracy is determined by $K$. When the interaction period is $0.2 \mathrm{~s}$ and $K_{x}=K_{y}=K_{z}=5$, this disturbance observer can reduce the disturbance tracking error to $13 \%$ in two interactions and to $1 \%$ in four interactions. Thus, for slowly timevarying external disturbances and continuous bounded model uncertainties, this disturbance observer has superior tracking performance.

\subsection{FNTSM-Based Electromagnetic Docking Controller} Design. Compared with other conventional control methods, $\mathrm{SMC}$ is a special kind of nonlinear control. The striking feature of SMC is its total insensitivity to system uncertainties and external disturbances. However, in conventional SMC, the sliding manifold is linear and the convergence of system states to the equilibrium point is asymptotical but not in finite time. TSM and NTSM introduce nonlinear terms to design the sliding manifolds and offer superior properties such as finite-time convergence and less steady-state errors. This paper presents a disturbance observer-based FNTSM controller for spacecraft electromagnetic docking. In this controller, global fast finite-time convergence is guaranteed, the singularity problem is avoided, a disturbance observer is adopted to estimate the model uncertainties and external disturbances, and a power reaching law is used to diminish the chattering.

The FNTSM manifold is designed as

$$
\boldsymbol{s}=\boldsymbol{e}+\boldsymbol{C}_{1} \boldsymbol{e}^{r}+\boldsymbol{C}_{2} \dot{\boldsymbol{e}}^{p / q},
$$

where $C_{1} \in R^{3 \times 3}$ and $C_{2} \in R^{3 \times 3}$ are diagonal matrices with positive elements; $r$ is a constant greater than $1 ; p$ and $q$ are positive odd numbers satisfying $q<p<2 q ; \boldsymbol{e}^{r}$ and $\dot{\boldsymbol{e}}^{p / q}$ are column vectors defined as $\boldsymbol{e}^{r}=\left[\begin{array}{lll}e_{x}^{r} & e_{y}^{r} & e_{z}^{r}\end{array}\right]^{\mathrm{T}}$ and $\dot{\boldsymbol{e}}^{p / q}=$ $\left[\begin{array}{lll}\dot{e}_{x}^{p / q} & \dot{e}_{y}^{p / q} & \dot{e}_{z}^{p / q}\end{array}\right]^{\mathrm{T}}$, respectively.

For convenient analysis, taking the scalar sliding manifold $s=0$ in Eq. (52) yields

$$
c_{2} \dot{e}^{p / q}=-e-c_{1} e^{r} .
$$

It is obvious that the convergence rate is governed by the linear term $-e$ near the equilibrium point and by the nonlinear term $-c_{1} e^{r}$ far from the equilibrium point, which guarantees global fast convergence.

To demonstrate the merits of FNTSM, the convergence rates of LSM and NTSM are compared.

$$
\mathrm{LSM}: c_{2} \dot{e}=-e \text {, }
$$

$$
\text { NTSM : } c_{2} \dot{e}^{p / q}=-e .
$$

The solutions of these two expressions are

$$
\begin{aligned}
e_{\mathrm{LSM}}(t) & =e_{\mathrm{LSM}}(0) \exp \left(-c_{2}^{-1} t\right) \\
e_{\mathrm{NTSM}}(t) & =\left(e_{\mathrm{NTSM}}(0)^{1-q / p}-(p-q) /\left(p c_{2}^{q / p}\right) \cdot t\right)^{1-q / p} .
\end{aligned}
$$

In Eq. (56), letting $e_{\mathrm{LSM}}(t)=0$ yields $t \longrightarrow \infty$.

In Eq. (57), letting $e_{\mathrm{NTSM}}(t)=0$ yields $t=e_{\mathrm{NTSM}}(0)^{1-q / p}$ $p c_{2}^{q / p} /(p-q)$.

Thus, the NTSM is a finite-time convergent, and the LSM is asymptotic convergent. Comparing Eq. (53) to Eq. (55), the FNTSM introduces a nonlinear term $-c_{1} e^{r}$, which results in faster convergence than that of the NTSM. Comparing Eq. (53) to Eq. (54), the number of the parameters of the FNTSM is more than that of the LSM, such as $p$ and $q$; thus, the convergence rate of the FNTSM can be adjusted more flexibly, and the FNTSM can converge to equilibrium point in finite time while the LSM cannot.

Thus, we choose the expression in Eq. (52) as the sliding manifold. Differentiating this sliding manifold $s$ yields

$$
\dot{\boldsymbol{s}}=\dot{\boldsymbol{e}}+r \boldsymbol{C}_{1} \operatorname{diag}\left(\boldsymbol{e}^{r-1}\right) \dot{\boldsymbol{e}}+\frac{p}{q} \boldsymbol{C}_{2} \operatorname{diag}\left(\dot{\boldsymbol{e}}^{p / q-1}\right) \ddot{\boldsymbol{e}}
$$

According to the docking dynamics model and the disturbance observer, the FNTSM controller is designed as

$$
\begin{aligned}
\boldsymbol{U}= & \boldsymbol{g}(\boldsymbol{\rho})^{-1}\left(-\boldsymbol{f}(\boldsymbol{\rho}, \dot{\boldsymbol{\rho}})-\widehat{\boldsymbol{D}}+\ddot{\boldsymbol{\rho}}_{d}-\frac{q}{p} \boldsymbol{C}_{2}^{-1} \operatorname{diag}\left(\dot{\boldsymbol{e}}^{2-p / q}\right)\right. \\
& \left.\cdot\left(\boldsymbol{I}_{3}+r \boldsymbol{C}_{1} \boldsymbol{e}^{r-1}\right)-\eta \operatorname{sgn}(\boldsymbol{s})-\vartheta \operatorname{diag}\left(|\boldsymbol{s}|^{\tau}\right) \operatorname{sgn}(\boldsymbol{s})\right),
\end{aligned}
$$

where $\boldsymbol{I}_{3} \in \boldsymbol{R}^{3 \times 1}$ denotes the unit column vector; $\eta>0, \vartheta>0$,

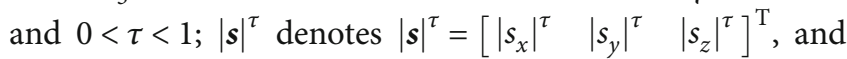
$\operatorname{sgn}(\boldsymbol{s})$ denotes the sign function.

Consider the following Lyapunov function

$$
V=\frac{1}{2} \boldsymbol{s}^{\mathrm{T}} \boldsymbol{s}+\frac{1}{2} \tilde{\boldsymbol{D}}^{\mathrm{T}} \tilde{\boldsymbol{D}} .
$$

Differentiating this expression yields

$$
\dot{V}=\boldsymbol{s}^{\mathrm{T}} \dot{\boldsymbol{s}}+\tilde{\boldsymbol{D}}^{\mathrm{T}} \dot{\tilde{\boldsymbol{D}}} .
$$


Substituting Eq. (58) and Eq. (50) into this expression yields

$$
\dot{V}=\boldsymbol{s}^{\mathrm{T}}\left(\dot{\boldsymbol{e}}+r \boldsymbol{C}_{1} \operatorname{diag}\left(\boldsymbol{e}^{r-1}\right) \dot{\boldsymbol{e}}+\frac{p}{q} \boldsymbol{C}_{2} \operatorname{diag}\left(\dot{\boldsymbol{e}}^{p / q-1}\right) \ddot{\boldsymbol{e}}\right)-\tilde{\boldsymbol{D}}^{\mathrm{T}} \boldsymbol{K} \tilde{\boldsymbol{D}} .
$$

Substituting Eq. (39) and Eq. (59) into this expression yields

$$
\begin{aligned}
\dot{V}= & \boldsymbol{s}^{\mathrm{T}}\left(\dot{\boldsymbol{e}}+r \boldsymbol{C}_{1} \operatorname{diag}\left(\boldsymbol{e}^{r-1}\right) \dot{\boldsymbol{e}}+\frac{p}{q} \boldsymbol{C}_{2} \operatorname{diag}\left(\dot{\boldsymbol{e}}^{\mid / q-1}\right)\left(\ddot{\boldsymbol{\rho}}-\ddot{\boldsymbol{\rho}}_{d}\right)\right)-\tilde{\boldsymbol{D}}^{\mathrm{T}} \boldsymbol{K} \tilde{\boldsymbol{D}} \\
= & \boldsymbol{s}^{\mathrm{T}}\left(\dot{\boldsymbol{e}}+r \boldsymbol{C}_{1} \operatorname{diag}\left(\boldsymbol{e}^{r-1}\right) \dot{\boldsymbol{e}}+\frac{p}{q} \boldsymbol{C}_{2} \operatorname{diag}\left(\dot{\boldsymbol{e}}^{p / q-1}\right)\left(\boldsymbol{f}(\boldsymbol{\rho}, \dot{\boldsymbol{\rho}})+\boldsymbol{g}(\boldsymbol{\rho}) \boldsymbol{U}+\boldsymbol{D}-\ddot{\boldsymbol{\rho}}_{d}\right)\right) \\
& -\tilde{\boldsymbol{D}}^{\mathrm{T}} \boldsymbol{K} \boldsymbol{D}=\boldsymbol{s}^{\mathrm{T}}\left(\frac{p}{q} \boldsymbol{C}_{2} \operatorname{diag}\left(\boldsymbol{e}^{p / q-1}\right)\left(\tilde{\boldsymbol{D}}-\eta \operatorname{sgn}(\boldsymbol{s})-9 \operatorname{diag}\left(|\boldsymbol{s}|^{\tau}\right) \operatorname{sgn}(\boldsymbol{s})\right)\right) \\
& -\tilde{\boldsymbol{D}}^{\mathrm{T}} \boldsymbol{K} \boldsymbol{D} .
\end{aligned}
$$

According to the definitions of the parameters in this expression, $p / q-1=(p-q) / q>0$ and the numerator $(p-$ $q)$ is an even number; thus, whatever the sign of $\dot{\boldsymbol{e}}$ is, we have $\dot{\boldsymbol{e}}^{p / q-1}>0$.

When $\boldsymbol{s}=0$, we have

$$
\dot{V}=-\tilde{\boldsymbol{D}}^{\mathrm{T}} \boldsymbol{K} \tilde{\boldsymbol{D}} \leq 0 .
$$

In this case, the system states are on the sliding manifold $\boldsymbol{s}=0$, and the states converge according to the designed sliding manifold.

When $\tilde{\boldsymbol{D}}=0$, we have

$$
\begin{aligned}
\dot{V} & =\boldsymbol{s}^{\mathrm{T}}\left(\frac{p}{q} \boldsymbol{C}_{2} \operatorname{diag}\left(\dot{\boldsymbol{e}}^{p / q-1}\right)\left(-\eta \operatorname{sgn}(\boldsymbol{s})-\vartheta \operatorname{diag}\left(|\boldsymbol{s}|^{\tau}\right) \operatorname{sgn}(\boldsymbol{s})\right)\right) \\
& <-\vartheta \frac{p}{q}|\boldsymbol{s}|^{\mathrm{T}} \boldsymbol{C}_{2} \operatorname{diag}\left(\dot{\mathrm{e}}^{p / q-1}\right)|\boldsymbol{s}|^{\tau}<0 .
\end{aligned}
$$

In this case, the system can be regarded as the FNTSM

\begin{tabular}{|c|c|}
\hline Parameters & Values \\
\hline Mass & Sat1: $2000 \mathrm{~kg}$; Sat2: $50 \mathrm{~kg}$ \\
\hline $\begin{array}{l}\text { Magnetic moment of } \\
\text { Sat1 }\end{array}$ & $500 \pi \mathrm{A} \cdot \mathrm{m}^{2}$ \\
\hline $\begin{array}{l}\text { Current amplitude of } \\
\text { Sat2 }\end{array}$ & $10 \mathrm{~A}$ \\
\hline Number of turns & 500 \\
\hline Orbit & $500 \mathrm{~km}$, circle orbit \\
\hline Direction of docking & $\begin{array}{c}\text { Along the velocity of the center of the } \\
\text { system }\end{array}$ \\
\hline Control cycle & $0.2 \mathrm{~s}$ \\
\hline Initial relative position & {$[0.05,2.00,-0.05]^{\mathrm{T}} \mathrm{m}$} \\
\hline Initial relative velocity & {$[-0.004,-0.001,0.005]^{\mathrm{T}} \mathrm{m} / \mathrm{s}$} \\
\hline External disturbances & $\begin{array}{c}x \text {-axis: } 10^{-4} \cos (0.05 \mathrm{t}) \mathrm{m} / \mathrm{s}^{2} \\
y \text {-axis: } 2 \times 10^{-4} \cos (0.05 t) \mathrm{m} / \mathrm{s}^{2} \\
z \text {-axis: }-2 \times 10^{-4} \sin (0.05 t) \mathrm{m} / \mathrm{s}^{2}\end{array}$ \\
\hline Controller parameters & $\begin{array}{c}C_{1}=\operatorname{diag}(1,1,1) \\
C_{2}=\operatorname{diag}(1000,1000,1000) \\
K=\operatorname{diag}(5,5,5) \\
p / q=5 / 3 ; r=5 / 3 \\
\vartheta=0.05 ; \tau=0.8\end{array}$ \\
\hline
\end{tabular}
control without external disturbances, and the states will converge to the sliding manifold $s=0$ according to the reaching law.

Further derivation of Eq. (63) yields

$$
\begin{aligned}
\dot{V}= & \boldsymbol{s}^{\mathrm{T}}\left(\frac{p}{q} \boldsymbol{C}_{2} \operatorname{diag}\left(\dot{\boldsymbol{e}}^{p / q-1}\right)\left(\tilde{\boldsymbol{D}}-\eta \operatorname{sgn}(\boldsymbol{s})-\vartheta \operatorname{diag}\left(|\boldsymbol{s}|^{\tau}\right) \operatorname{sgn}(\boldsymbol{s})\right)\right) \\
& -\tilde{\boldsymbol{D}}^{\mathrm{T}} \boldsymbol{K} \boldsymbol{D}=\frac{p}{q} \boldsymbol{s}^{T} \boldsymbol{C}_{2} \operatorname{diag}\left(\dot{\boldsymbol{e}}^{p / q-1}\right)(\tilde{\boldsymbol{D}}-\eta \operatorname{sgn}(\boldsymbol{s})) \\
& -\vartheta \frac{p}{q}|\boldsymbol{s}|^{\mathrm{T}} \boldsymbol{C}_{2} \operatorname{diag}\left(\dot{\boldsymbol{e}}^{p / q-1}\right)|\boldsymbol{s}|^{\tau}-\tilde{\boldsymbol{D}}^{\mathrm{T}} \boldsymbol{K} \boldsymbol{D} \\
\leq & \frac{p}{q}|\boldsymbol{s}|^{\mathrm{T}} \boldsymbol{C}_{2} \operatorname{diag}\left(\dot{\boldsymbol{e}}^{p / q-1}\right)\left(|\tilde{\boldsymbol{D}}|-\eta \boldsymbol{I}_{3}\right)-\vartheta \frac{p}{q}|\boldsymbol{s}|^{\mathrm{T}} \boldsymbol{C}_{2} \operatorname{diag}\left(\dot{\boldsymbol{e}}^{p / q-1}\right)|\boldsymbol{s}|^{\tau} \\
& -\tilde{\boldsymbol{D}}^{\mathrm{T}} \boldsymbol{K} \boldsymbol{D}=\frac{p}{q}|\boldsymbol{s}|^{\mathrm{T}} \boldsymbol{C}_{2} \operatorname{diag}\left(\dot{\boldsymbol{e}}^{p / q-1}\right)\left(|\tilde{\boldsymbol{D}}|-\eta \boldsymbol{I}_{3}-\vartheta|\boldsymbol{s}|^{\tau}\right)-\tilde{\boldsymbol{D}}^{\mathrm{T}} \boldsymbol{K} \boldsymbol{D} .
\end{aligned}
$$

TABLE 1: Simulation parameters.

The sign of this expression is determined by the term $\left(|\tilde{\boldsymbol{D}}|-\eta \boldsymbol{I}_{3}-\vartheta|\boldsymbol{s}|^{\tau}\right)$. Suppose the initial estimation error of the disturbance observer $\tilde{\boldsymbol{D}}$ is $\tilde{\boldsymbol{D}}(0)$ and $\tilde{\boldsymbol{D}}$ converges to zero exponentially, as in Eq. (51). We take $\eta$ as a positive constant greater than the largest element in $|\tilde{\boldsymbol{D}}(0)|$. Thus, we have

$$
\dot{V} \leq-\vartheta \frac{p}{q}|\boldsymbol{s}|^{T} \boldsymbol{C}_{2} \operatorname{diag}\left(\dot{\boldsymbol{e}}^{p / q-1}\right)|\boldsymbol{s}|^{\tau}-\tilde{\boldsymbol{D}}^{\mathrm{T}} \boldsymbol{K} \boldsymbol{D} \leq 0
$$

Therefore, the system is stable.

4.4. Docking Trajectory. To take the controllability of the system into account and avoid excessive control gains, predesigning a time-driven desired trajectory to provide docking guidance may be advisable.

In this paper, the desired trajectory is designed by the expressions in Eq. (68). The advantage of this kind of trajectory is the nonlinear relationship between relative distance and relative velocity, which accords with the characteristics of the electromagnetic force. To increase docking efficiency and reduce time consumption, the docking trajectory designed in this paper has three phases: the acceleration phase, the deceleration phase, and the constant velocity phase. These phases are defined as follows:

$\dot{\rho}_{d}= \begin{cases}\left(\dot{\rho}_{1}-\dot{\rho}_{0}\right) /\left(\rho_{1}-\rho_{0}\right) \cdot\left(\rho_{d}-\rho_{0}\right)+\dot{\rho}_{0} & , \rho_{d}>\rho_{1} \\ \left(\dot{\rho}_{2}-\dot{\rho}_{1}\right) /\left(\rho_{2}-\rho_{1}\right) \cdot\left(\rho_{d}-\rho_{2}\right)+\dot{\rho}_{2} & , \rho_{2}<\rho_{d} \leq \rho_{1}, \\ \dot{\rho}_{2} \quad, \rho_{d} \leq \rho_{2} & \end{cases}$ 


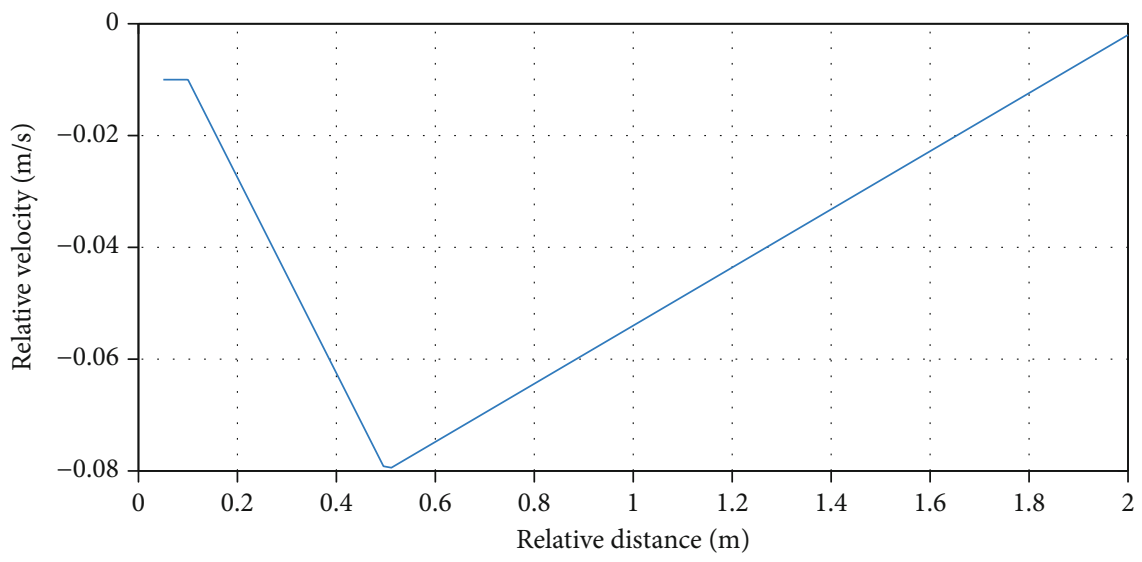

Figure 12: Docking trajectory design.

where $\rho_{d}$ and $\dot{\rho}_{d}$ are the relative distance with respect to the initial value $\rho_{0}$ and the relative velocity with respect to the initial value $\dot{\rho}_{0}$, respectively; $\rho_{i}(i=1,2)$ and $\dot{\rho}_{i}(i=1,2)$ are the switching parameters of the relative distance and relative velocity. With the predesign of $\rho_{i}(i=0,1,2)$ and $\dot{\rho}_{i}(i=0,1$, $2)$, the desired relative distance and velocity driven by time are obtained.

\section{Numerical Simulations and Analyses}

In this section, numerical simulations are conducted using the proposed control scheme. The control commands are computed by the presented controller, and the actual control variables are computed by the exact model.

5.1. Simulation Parameters. The simulation parameters are given in Table 1.

For the docking trajectory, we take $\rho_{0}=2 \mathrm{~m}, \rho_{1}=0.5 \mathrm{~m}$, $\rho_{2}=0.1 \mathrm{~m}, \dot{\rho}_{0}=-0.002 \mathrm{~m} / \mathrm{s}, \dot{\rho}_{1}=0.08 \mathrm{~m} / \mathrm{s}$, and $\dot{\rho}_{2}=-0.01$ $\mathrm{m} / \mathrm{s}$ in the direction of docking, and the desired relative positions and desired relative velocities in the other two directions are all taken to be 0 . To avoid the singularity of the trajectory solution, the initial desired relative velocity cannot be 0 but it can be a small number. The designed docking trajectory is shown in Figure 12.

5.2. Simulation Results. In this section, two simulation scenarios are presented to illustrate the necessity of modifying the far-field model and the effectiveness of the proposed control scheme.

Scenario 1 . Verification of the necessity of modifying the far-field model without external disturbances.

In this scenario, three simulations are conducted and compared with each other to verify the necessity of modifying the far-field model. Here, to make the verification convincing, the unknown external disturbances are not considered. The three simulations are (1) no modification of the far-field model and no use of the disturbance observer (nonmodification and non-DO), (2) no modification of the far-field model and use of the disturbance observer (nonmodification and DO), and (3) modification of the far-field model and use of the disturbance observer (modification and DO).

Figure 13 shows the variations of the sliding manifold components relative to time. Figure 14 shows the variations of the position tracking errors and velocity tracking errors relative to time. Figure 15 shows the variations of the desired control forces and actual control forces relative to time. In the case of "nonmodification and non-DO", the model error increases rapidly with decreasing relative distance, and the actual forces acting on the satellites become smaller than the calculated desired control forces in the final phase (see Figure 15). Thus, the state components of the $x$-axis and the $y$-axis cannot be maintained on the sliding manifolds (see Figure 13), which results in increased tracking errors (see Figure 14). In the case of "nonmodification and DO," although the results improve, the model error increases so fast that the disturbance observer is unable to track it in time. In the case of "modification and DO," the simulation results show the best performances among these three cases because the model error is restricted to a slowly changing and smallbounded range and can be estimated and compensated effectively by the disturbance observer. It should be noted that the components of the $z$-axis converge to 0 in all three cases, since the model is more accurate in the early phase and the motions of the $z$-axis are decoupled from the $x$-axis and the $y$-axis.

From the simulation results above, in order to design a robust and high-precision controller for electromagnetic docking, it is important and necessary to modify the farfield model for establishing the dynamics model.

Scenario 2. Electromagnetic docking control with model uncertainties and external disturbances.

In this scenario, the improved far-field model, disturbance observer, and FNTSM controller are combined and the model uncertainties and unknown external disturbances are considered, and a simulation is conducted to verify the effectiveness of the proposed control scheme.

Figure 16 shows the variations of the relative positions during docking in a three-dimensional representation. It can be seen from this figure that along the docking direction ( $y$-axis), there exist relative position deviations on the $x$-axis 

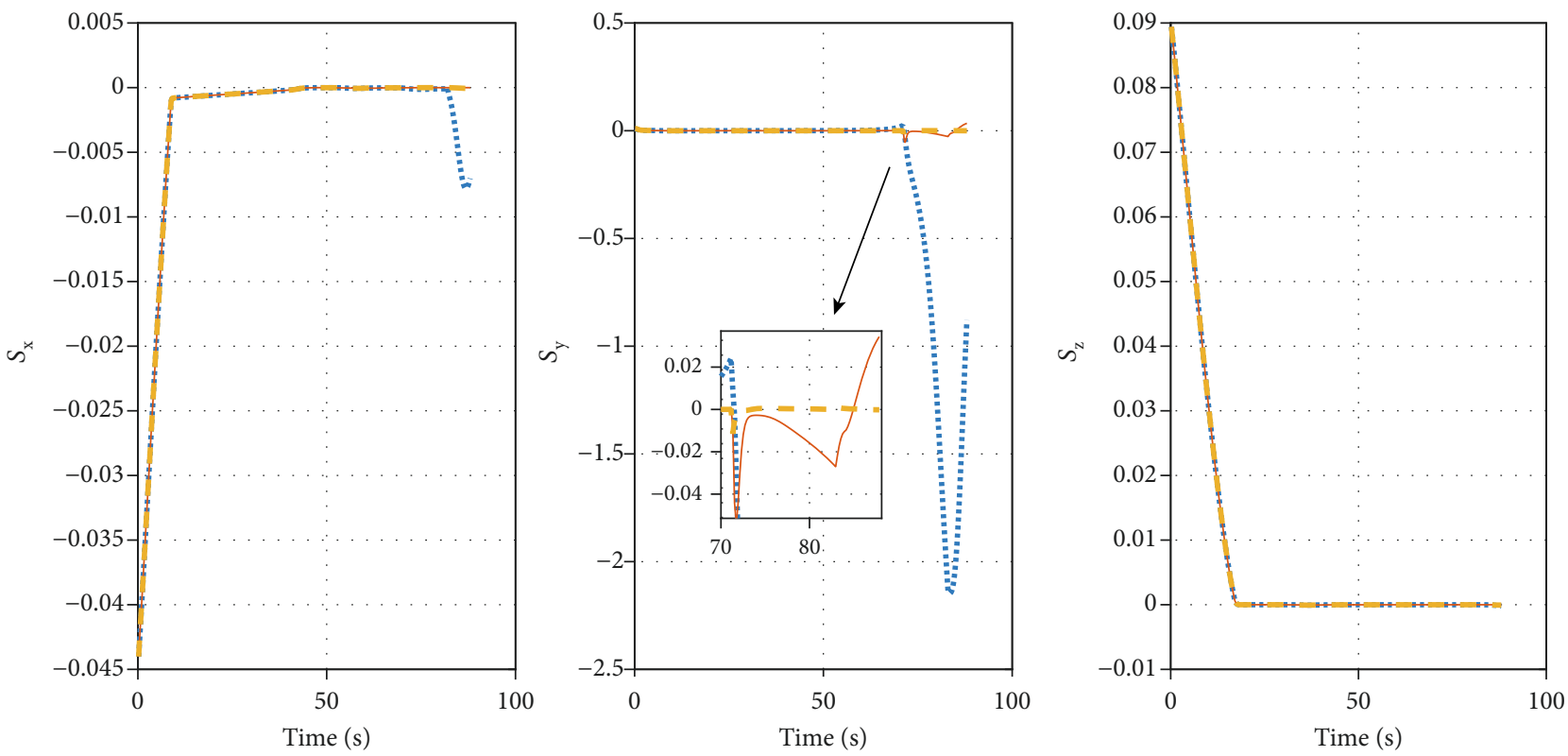

Nonmodification and non-DO

Nonmodification and DO

Modification and DO

FIGURE 13: Comparisons of the sliding manifold components.
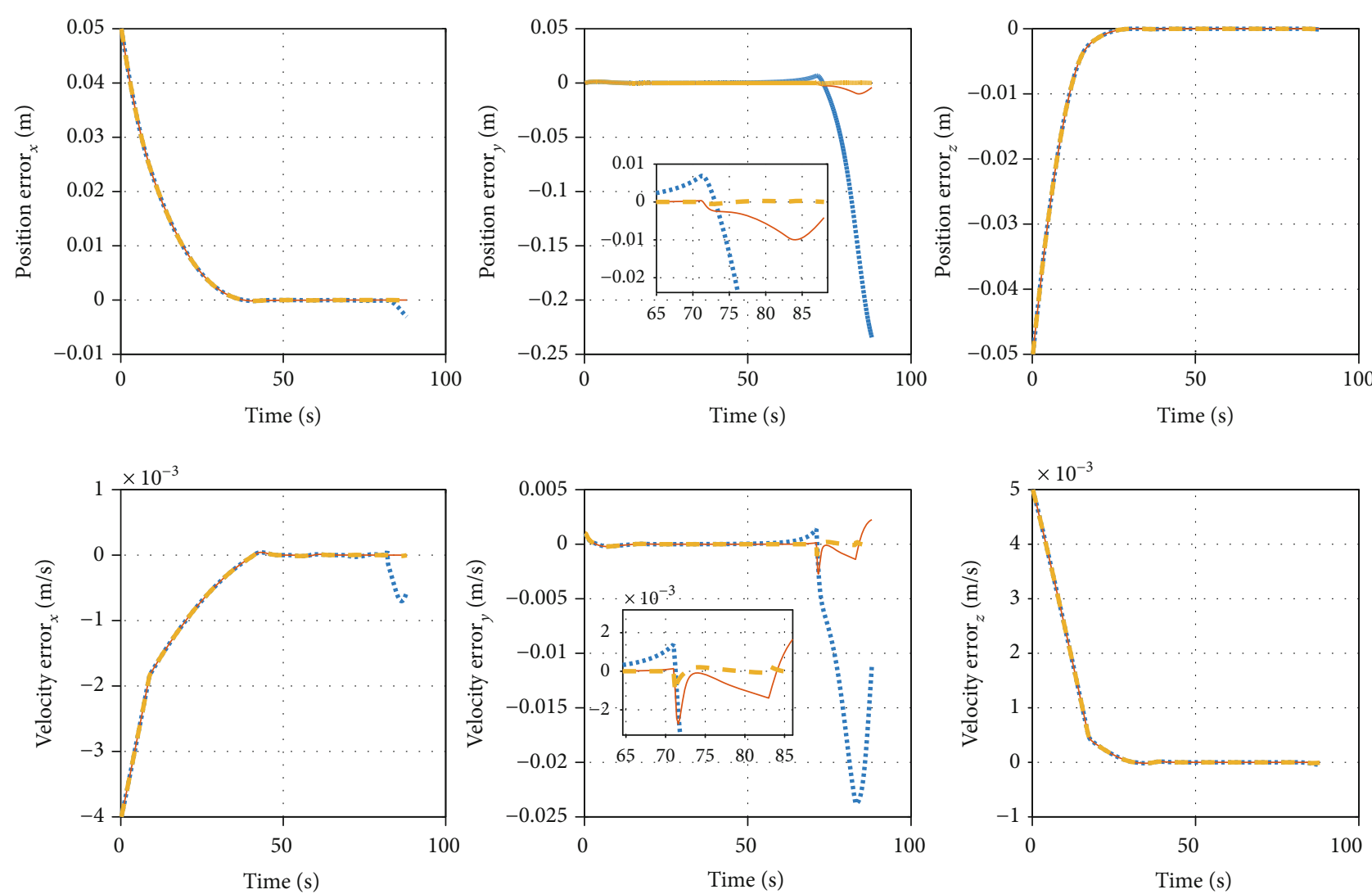

Nonmodification and non-DO

Nonmodification and DO

Modification and DO

FIGURE 14: Comparisons of the trajectory tracking error. 

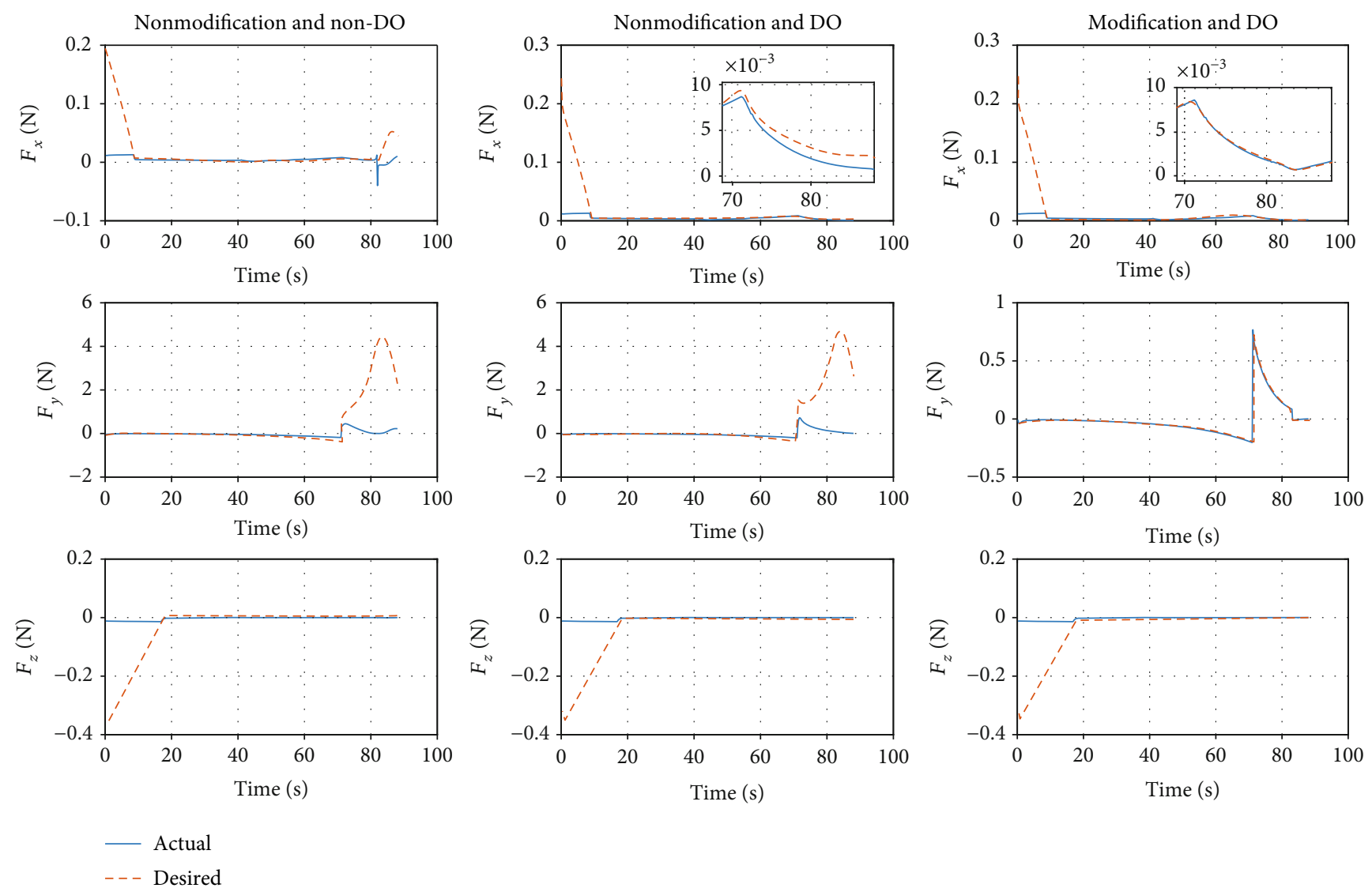

FIGURE 15: Comparisons of the desired control forces and actual control forces.
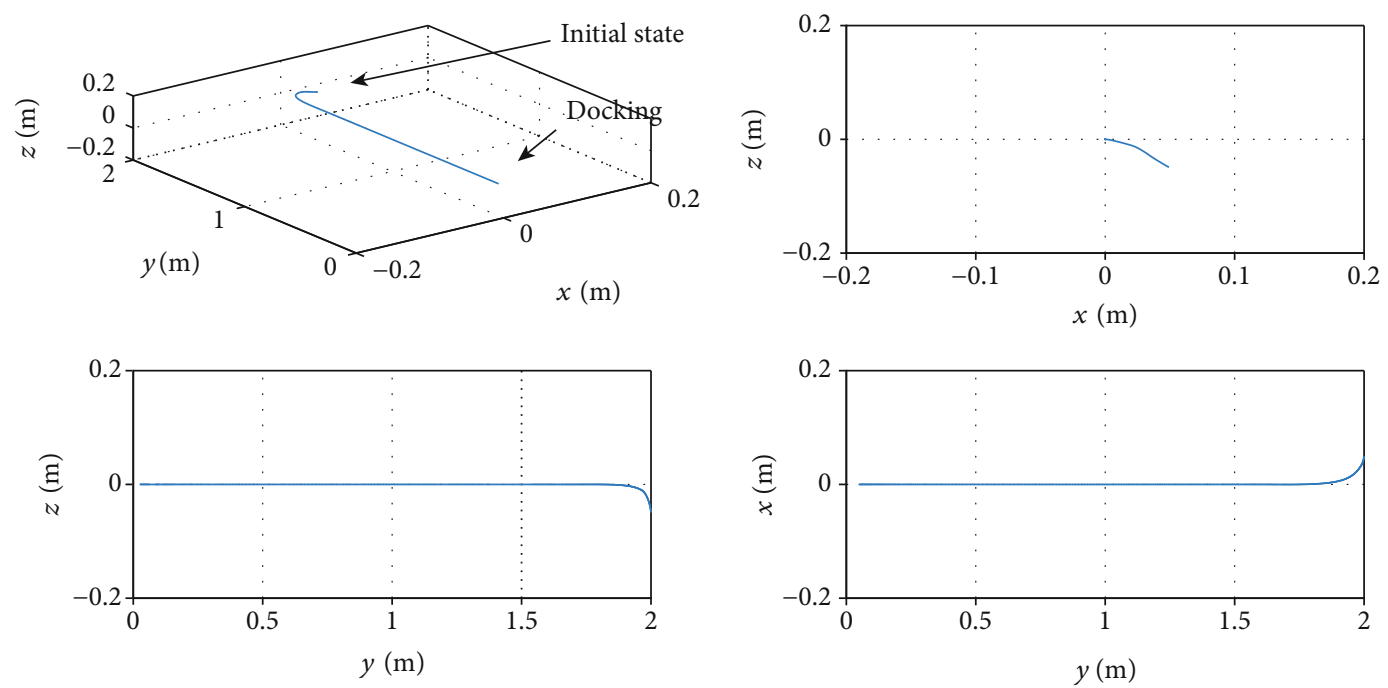

Figure 16: Three-dimensional representation and projections of relative positions.

and the $z$-axis at the initial time, and these deviations are eliminated at the relative distance of $1.8 \mathrm{~m}$.

Figure 17 shows the variations of the relative position components and relative velocity components relative to time. In the improved far-field model, the modification terms can on-line self-adjust and directly influence the control input by modifying the current on each coil to make the actual forces correspond to the desired forces. It can be seen from Figure 17 that in the presence of the initial state deviations, two spacecraft can realize soft-docking using the proposed control scheme. The docking takes $84 \mathrm{~s}$, and the relative velocity is $0.01 \mathrm{~m} / \mathrm{s}$ in the end.

Figure 18 shows the variations of the sliding manifold components relative to time. Figure 19 shows the variations 

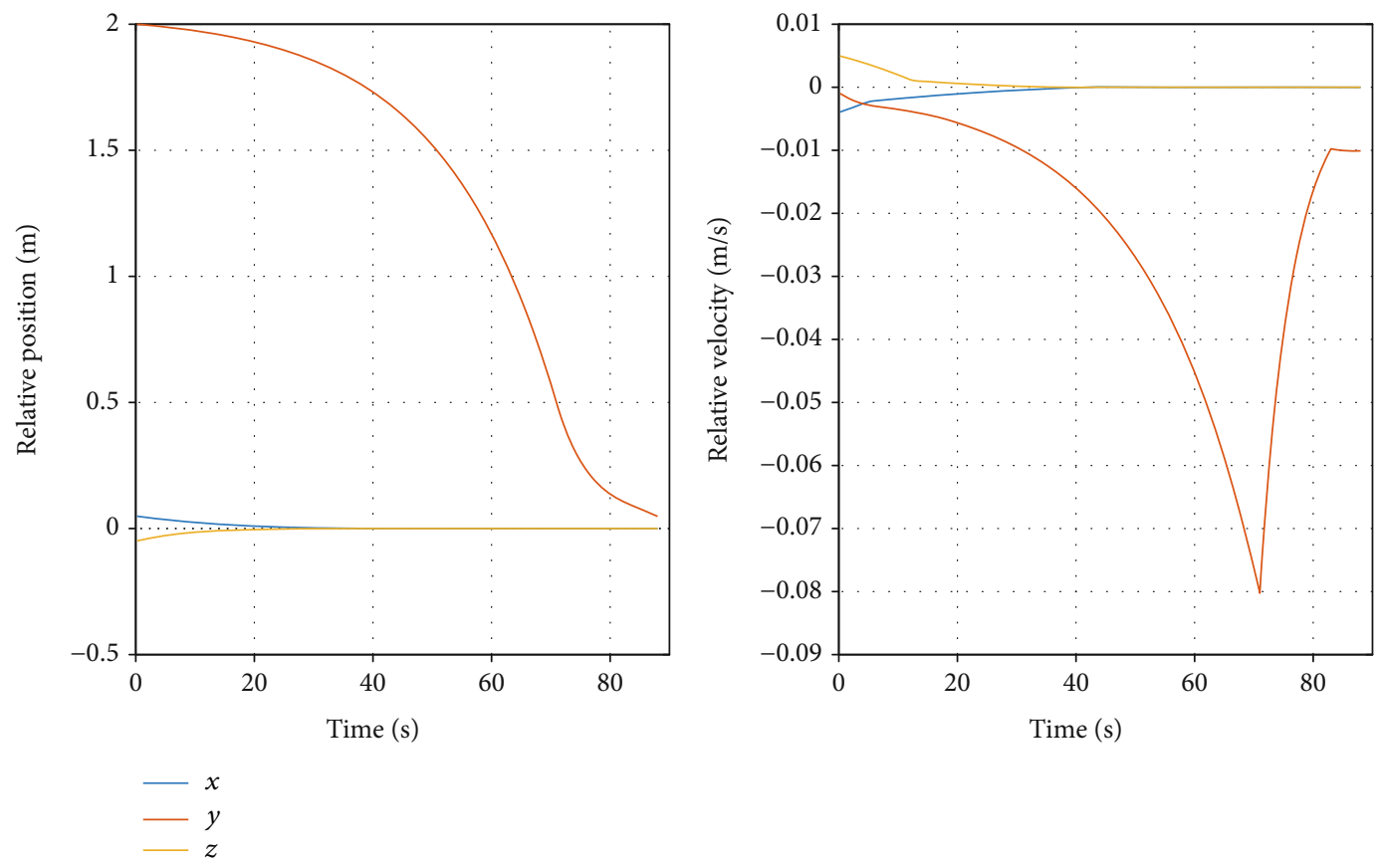

FIGURE 17: Relative position components and relative velocity components.

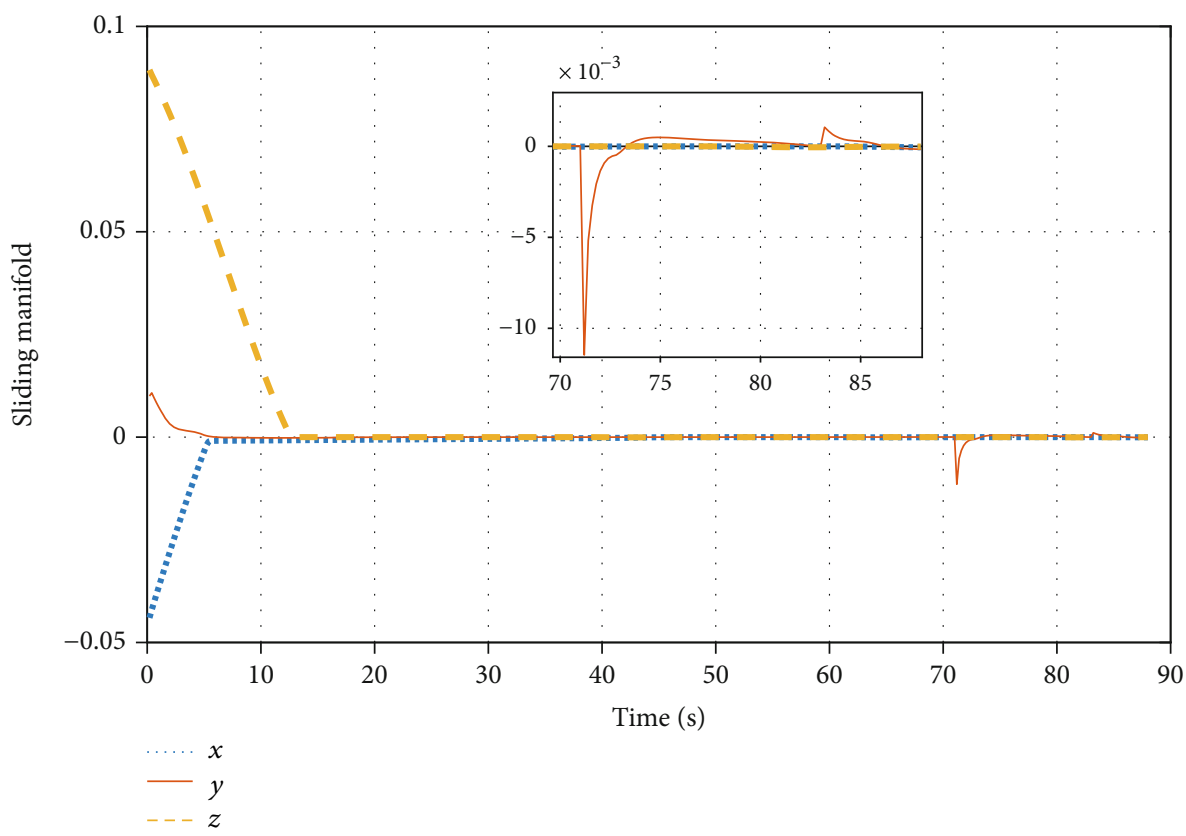

FIGURE 18: Components of the sliding manifold.

of the currents on the coils of Sat 2 relative to time. At the beginning of docking, the states starting off the sliding manifold $s=0$ move toward it, and the chattering is diminished, which means that the adopted reaching law is effective and reasonable. During the reaching phase of each axis, the current on the corresponding coil is saturated (see Figure 19), which means that the states are driven by the controller to move to the sliding manifold $s=0$ as fast as possible. Follow- ing the sliding phase, the states are confined to the sliding manifold $s=0$ and move according to Eq. (53).

Figure 20 shows the variations of the position tracking error components and velocity tracking error components relative to time. For the motions on the $x$-axis and $z$-axis, since the desired relative positions and desired relative velocities are all 0 , the initial tracking errors are the initial deviations along the docking direction. It can be seen that 


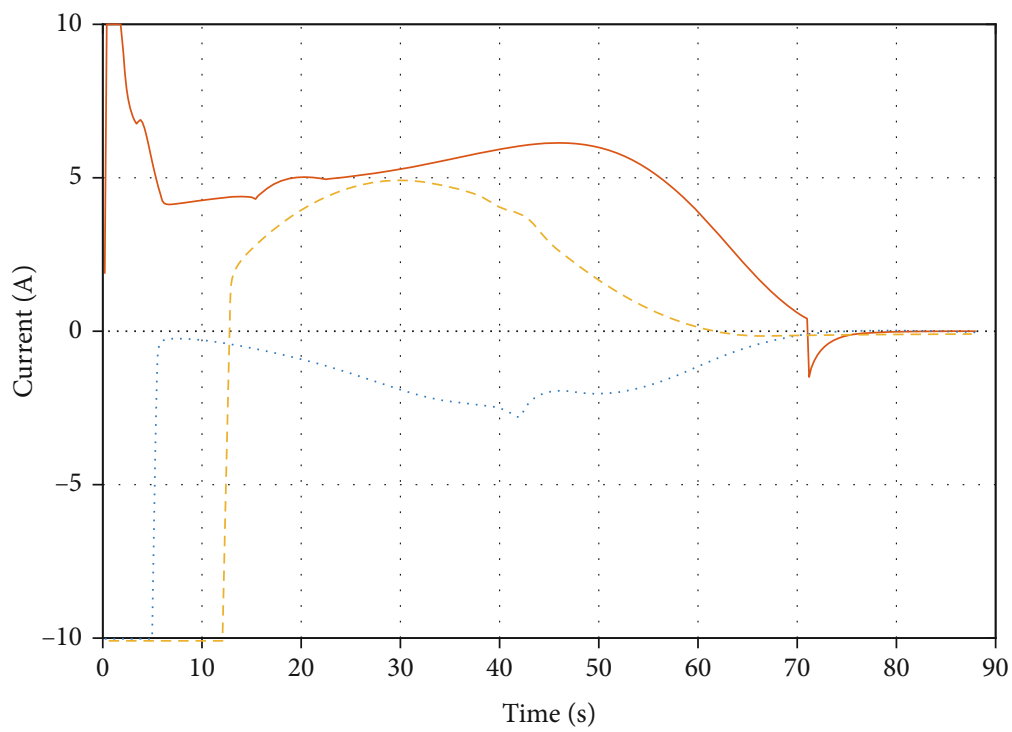

Coil3 $(x)$

Coil2 $(y)$

Coil4 $(z)$

FIgURE 19: Currents on the coils of Sat2.
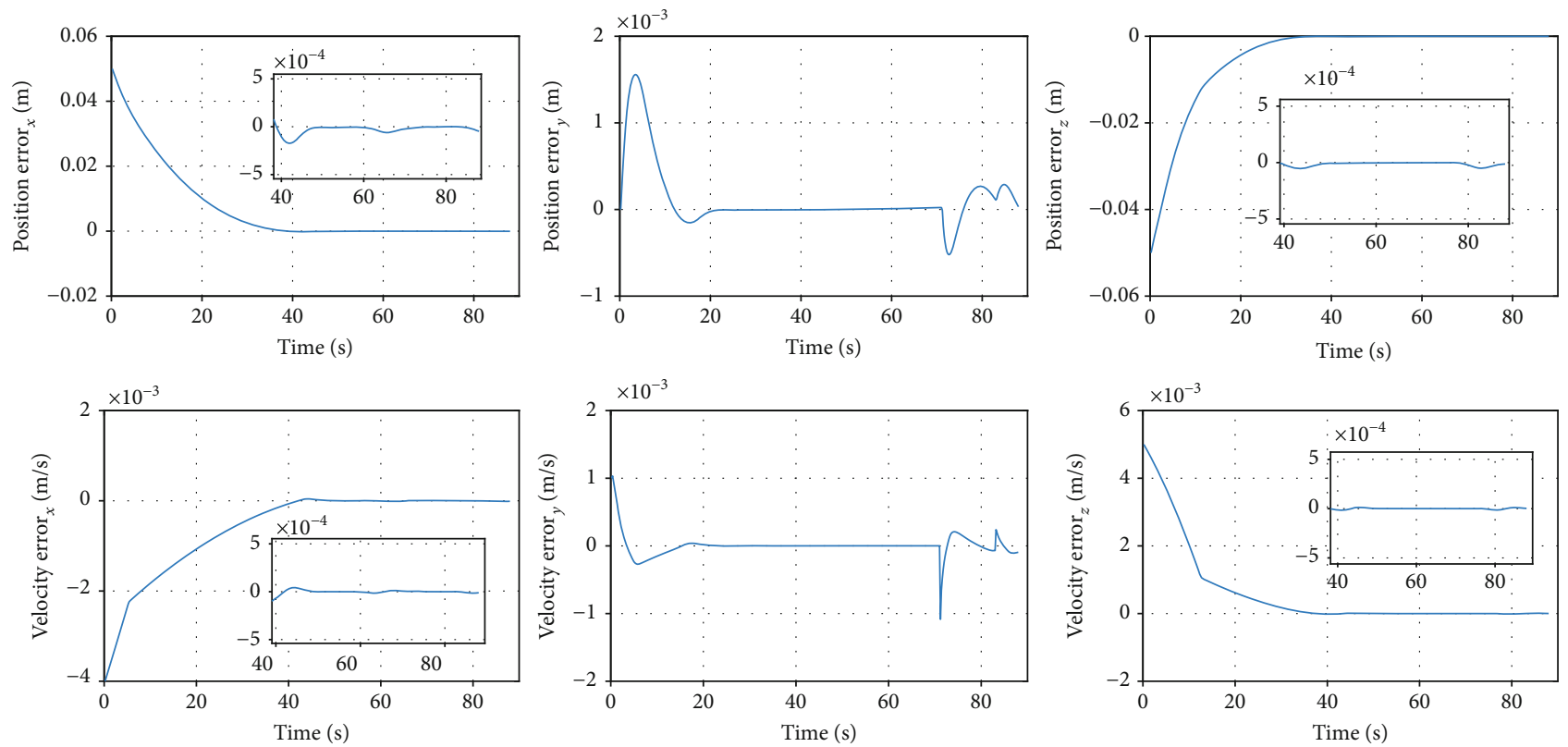

FIGURE 20: Position tracking errors and velocity tracking errors.

the tracking errors on the $x$-axis and the $z$-axis converge to 0 at $45 \mathrm{~s}$ and that after $45 \mathrm{~s}$, the motions on the $x$-axis and the $z$-axis are not uncontrolled (see Figure 19) because of the external disturbances and the dynamics coupling of the $x$-axis and the $y$-axis (as in Eq. (17)). On the $y$-axis, the motions are driven by the designed docking trajectory. The position tracking accuracy and the velocity tracking accuracy are superior to $1 \mathrm{~mm}$ and $1 \mathrm{~mm} / \mathrm{s}$, respectively, which verifies that the proposed control scheme provides a fast convergence rate and high tracking accuracy.

For the proposed disturbance observer, Figure 21 shows the comparison of the actual disturbances and the estimated disturbances and the variations of the tracking errors relative to time. It should be pointed out that the actual disturbances shown in this figure consist of the model uncertainties and unknown external disturbances, and the sample cycle of the 

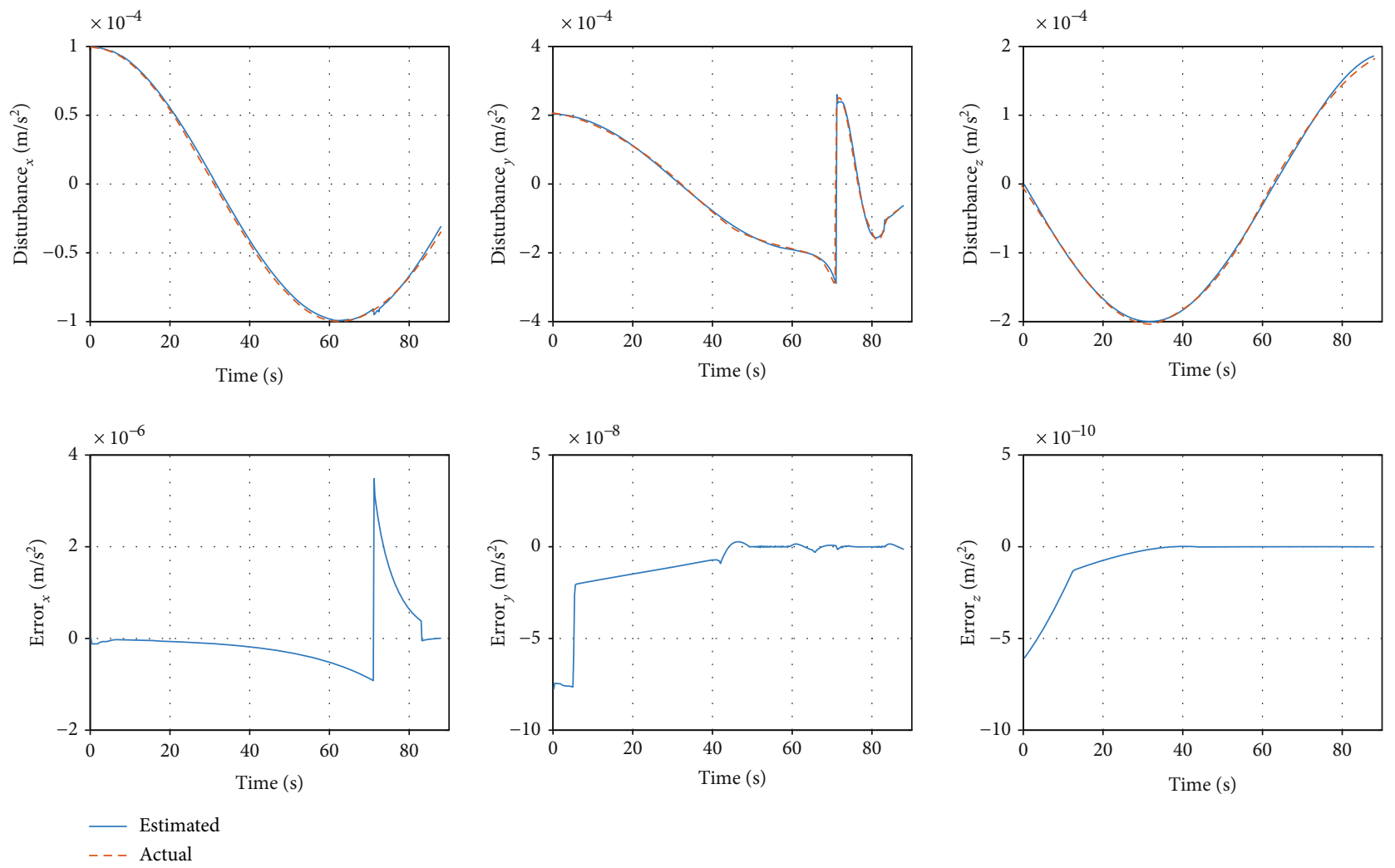

FIgURE 21: Tracking results of model uncertainties and unknown external disturbances.

observer is equal to the control cycle of $0.2 \mathrm{~s}$. It can be seen that the tracking rate is fast and high-precision tracking can be guaranteed.

\section{Conclusions}

This paper presents a spacecraft electromagnetic docking control scheme combining an improved far-field model, a fast-nonsingular terminal sliding mode controller, and a disturbance observer. In contrast with electromagnetic formation flight, it is feasible to use normal magnetic coils rather than superconducting coils to realize spacecraft electromagnetic docking because of the close range of the docking process. Because the error of the far-field model increases with decreasing relative distance, the improved far-field model is proposed, and the percent error is reduced within $5 \%$ when the axes of two coils are aligned. The modification terms of the improved model can online self-adjust in terms of the system states. Taking energy consumption as an index, the magnetic moment allocation problem is analyzed. When the magnetic moment of one spacecraft points to another, the calculated energy consumption of the other spacecraft is minimized in terms of the desired electromagnetic force. A disturbance observerbased fast-nonsingular terminal sliding mode controller for electromagnetic docking is designed, which offers not only the main advantages of linear sliding model control and nonsingular terminal sliding model control but also a global fast finite-time convergence rate, and the stability of the closed-loop system is proven. Two simulation scenarios are conducted: the first one verifies the necessity of modifying the far-field model, and the second one confirms the superior features of the proposed control scheme, such as global fast finite-time convergence, strong robustness, high-precision control, and chattering-free property.

\section{Data Availability}

No data were used to support this study.

\section{Conflicts of Interest}

The authors declare that there is no conflict of interest regarding the publication of this paper.

\section{References}

[1] L. Ge, B. Burkhart, and R. W. De Doncker, "Fast iron loss and thermal prediction method for power density and efficiency improvement in switched reluctance machines," IEEE Transactions on Industrial Electronics, vol. 67, no. 6, pp. 4463-4473, 2019.

[2] S. A. Schweighart and R. J. Sedwick, "Explicit dipole trajectory solution for electromagnetically controlled spacecraft clusters," Journal of Guidance, Control, and Dynamics, vol. 33, no. 4, pp. 1225-1235, 2010.

[3] C. Liu, K. Shi, X. Yue, and Z. Sun, "Inertia-freesaturated output feedback attitude stabilization for uncertain spacecraft," 
International Journal of Robust and Nonlinear Control, vol. 30, no. 13, pp. 5101-5121, 2020.

[4] J. D. Biggs and G. Livornese, "Control of a thrust-vectoring CubeSat using a single variable-speed control moment gyroscope," Journal of Guidance, Control, and Dynamics, vol. 43, no. 10, pp. 1865-1880, 2020.

[5] C. Liu, X. Yue, K. Shi, and Z. Sun, "Inertia-free attitude stabilization for flexible spacecraft with active vibration suppression," International Journal of Robust and Nonlinear Control, vol. 29, no. 18, pp. 6311-6336, 2019.

[6] R. C. Foust, E. S. Lupu, Y. K. Nakka, S. J. Chung, and F. Y. Hadaegh, "Ultra-soft electromagnetic docking with applications to in-orbit assembly," in 69th International Astronautical Congress, Bremen, Germany, 2018.

[7] A. Torisaka, S. Ozawa, H. Yamakawa, and N. Kobayashi, "Position and Attitude Control of Formation Flying Satellites for Restructuring their Configurations by Electromagnetic Forces at the Docking Phase," in AIAA Guidance, Navigation, and Control Conference, 2013.

[8] G. Zeng and M. Hu, "Finite-time control for electromagnetic satellite formations," Acta Astronautica, vol. 74, pp. 120-130, 2012.

[9] U. Ahsun, D. W. Miller, and J. L. Ramirez, "Control of electromagnetic satellite formations in near-earth orbits," Journal of Guidance, Control, and Dynamics, vol. 33, no. 6, pp. 18831891, 2010.

[10] A. K. Porter, D. J. Alinger, R. J. Sedwick et al., "Demonstration of electromagnetic formation flight and wireless power transfer," Journal of Spacecraft and Rockets, vol. 51, no. 6, pp. 1914-1923, 2014.

[11] K. W. Yung, P. B. Landecker, and D. D. Villani, "An analytic solution for the force between two magnetic dipoles," Magnetic and Electrical Separation, vol. 9, no. 1, pp. 39-52, 1998.

[12] P. B. Landecker, D. D. Villani, and K. W. Yung, "An analytic solution for the torque between two magnetic dipoles," Magnetic and Electrical Separation, vol. 10, no. 1, pp. 29-33, 1999.

[13] S. A. Schweighart, "Electromagnetic formation flight dipole solution planning," Ph.D. Thesis, Massachusetts Institute of Technology, Cambridge, MA, 2005.

[14] R. Ravaud, G. Lemarquand, S. Babic, V. Lemarquand, and C. Akyel, "Cylindrical magnets and coils: fields, forces, and inductances," IEEE Transactions on Magnetics, vol. 46, no. 9, pp. 3585-3590, 2010.

[15] W. Robertson, B. Cazzolato, and A. Zander, "A simplified force equation for coaxial cylindrical magnets and thin coils," IEEE Transactions on Magnetics, vol. 47, pp. 2045-2049, 2010.

[16] J. T. Conway, "Forces between thin coils with parallel axes using Bessel functions," IEEE Transactions on Magnetics, vol. 49, no. 9, pp. 5028-5034, 2013.

[17] D. W. Kwon, "Propellantless formation flight applications using electromagnetic satellite formations," Acta Astronautica, vol. 67, no. 9-10, pp. 1189-1201, 2010.

[18] Y. W. Zhang, L. P. Yang, Y. W. Zhu, and H. Huang, "Dynamics and solutions for multispacecraft electromagnetic orbit correction," Journal of Guidance, Control, and Dynamics, vol. 37, no. 5, pp. 1604-1610, 2014.

[19] B. Z. Reinhardt and M. A. Peck, "New electromagnetic actuator for on-orbit inspection," Journal of Spacecraft and Rockets, vol. 53, no. 2, pp. 241-248, 2016.
[20] J. Zhang, J. D. Biggs, D. Ye, and Z. Sun, "Finite-time attitude set-point tracking for thrust-vectoring spacecraft rendezvous," Aerospace Science and Technology, vol. 96, p. 105588, 2020.

[21] C. Liu, Z. Sun, K. Shi, and F. Wang, "Robust dynamic output feedback control for attitude stabilization of spacecraft with nonlinear perturbations," Aerospace Science and Technology, vol. 64, pp. 102-121, 2017.

[22] D. Petrillo, M. Buonomo, A. Cavinato et al., "Flexible electromagnetic leash docking system (FELDs) experiment from design to microgravity testing," in 66th International Astronautical Congress, Jerusalem, Israel, 2015.

[23] Y. Geng, C. Li, Y. Guo, and J. D. Biggs, "Hybrid robust and optimal control for pointing a staring-mode spacecraft," Aerospace Science and Technology, vol. 105, 2020.

[24] K. Shi, C. Liu, and Z. Sun, "Constrained fuel-free control for spacecraft electromagnetic docking in elliptical orbits," Acta Astronautica, vol. 162, pp. 14-24, 2019.

[25] K. Shi, C. Liu, Z. Sun, and X. Yue, "Disturbance observer-based attitude stabilization for rigid spacecraft with input MRCs," Advances in Space Research, vol. 66, no. 3, pp. 689-701, 2020.

[26] K. Shi, C. Liu, J. D. Biggs, Z. Sun, and X. Yue, "Observer-based control for spacecraft electromagnetic docking," Aerospace Science and Technology, vol. 99, 2020.

[27] Y. Zhang, L. P. Yang, Y. W. Zhu, D. W. Qi, and Y. P. Li, "Magnetic capture volume derivation and multi-magnetic dipoles configuration design for spacecraft soft-docking," Advances in Space Research, vol. 63, no. 8, pp. 2399-2409, 2019.

[28] C. Liu, G. Vukovich, Z. Sun, and K. Shi, "Observer-based fault-tolerant attitude control for spacecraft with input delay," Journal of Guidance, Control, and Dynamics, vol. 41, no. 9, pp. 2041-2053, 2018.

[29] C. Liu, K. Shi, and Z. Sun, "Spacecraft chaotic attitude control with certain actuator failure based on integral sliding mode," Journal of Engineering Research, vol. 6, no. 1, pp. 153-166, 2018.

[30] J. Zhang, G. Zeng, Y. Gao, and J. Wang, "Underactuated microsatellite electromagnetic docking control using disturbance observer-based sliding mode controller," IEEE Access, vol. 8, pp. 124476-124485, 2020.

[31] J. Zhang, D. Ye, J. D. Biggs, and Z. Sun, "Finite-time relative orbit-attitude tracking control for multi-spacecraft with collision avoidance and changing network topologies," Advances in Space Research, vol. 63, no. 3, pp. 1161-1175, 2018.

[32] J. Zhang, D. Ye, Z. Sun, and C. Liu, "Extended state observer based robust adaptive control on $\mathrm{SE}(3)$ for coupled spacecraft tracking maneuver with actuator saturation and misalignment," Acta Astronautica, vol. 143, pp. 221-233, 2018.

[33] M. Boukattaya, N. Mezghani, and T. Damak, "Adaptive nonsingular fast terminal sliding-mode control for the tracking problem of uncertain dynamical systems," ISA Transactions, vol. 77, pp. 1-19, 2018.

[34] J. Zhang, J. D. Biggs, D. Ye, and Z. Sun, "Extended-stateobserver-based event-triggered orbit-attitude tracking for low-thrust spacecraft," IEEE Transactions on Aerospace and Electronic Systems, vol. 56, no. 4, pp. 2872-2883, 2020. 